\title{
Form-factors in the Baxter-Bazhanov-Stroganov model I: Norms and matrix elements
}

\author{
G von Gehlen ${ }^{\dagger}$, N Iorgov ${ }^{\ddagger}$, S Pakuliak ${ }^{\sharp}$, V Shadura ${ }^{\ddagger}$ and \\ Yu Tykhyy \\ † Physikalisches Institut der Universität Bonn, Nussallee 12, D-53115 Bonn, Germany \\ ¥ Bogolyubov Institute for Theoretical Physics, Kiev 03680, Ukraine \\ \# Bogoliubov Laboratory of Theoretical Physics, Joint Institute for Nuclear Research, \\ Dubna 141980, Moscow region, Russia \\ b Institute of Theoretical and Experimental Physics, Moscow 117259, Russia \\ E-mail: gehlen@th.physik.uni-bonn.de, iorgov@bitp.kiev.ua, \\ pakuliak@theor.jinr.ru, shadura@bitp.kiev.ua, tykhyy@bitp.kiev.ua
}

\begin{abstract}
We continue our investigation of the $\mathbb{Z}_{N}$-Baxter-Bazhanov-Stroganov model using the method of separation of variables [1]. In this paper we calculate the norms and matrix elements of a local $\mathbb{Z}_{N}$-spin operator between eigenvectors of the auxiliary problem. For the norm the multiple sums over the intermediate states are performed explicitly. In the case $N=2$ we solve the Baxter equation and obtain form-factors of the spin operator of the periodic Ising model on a finite lattice.
\end{abstract}

25 October $2018 \quad$ Submitted to: J. Phys. A: Math. Gen.

PACS numbers: $75.10 \mathrm{Hk}, 75.10 \mathrm{Jm}, 05.50+\mathrm{q}, 02.30 \mathrm{Ik}$

\section{Introduction}

The Baxter-Bazhanov-Stroganov model (BBS-model, also known as the $\tau^{(2)}$-model) [2, 3, 4, 5] has attracted considerable interest, because via functional relations it is related to the solvable $\mathbb{Z}_{N}$-chiral Potts model (CPM). Solving these functional relations has been the main method to calculate the eigenvalues of the CPM [6]. However, also by itself, the BBS-model is an interesting lattice spin model with cyclic $\mathbb{Z}_{N}$ spin-variables, which is closely related to the six-vertex model at roots of unity [7, 4, 8]. The BBS model in its vertex formulation can be solved using the Functional Bethe ansatz or Separation of Variables (SoV) method [9]. For $N=2$ it is equivalent to a generalized free-fermion Ising model [10].

Using the formulation of the BBS-model in terms of cyclic $L$-operators [7, 4, 11], in [1] we developed a general method for the construction of its transfer matrix eigenvectors. Our approach is an adaptation of the $\mathrm{SoV}$ method of [9, 12] to $N$-state spin chain models. In order to find the eigenvectors of the transfer matrix we first obtain the eigenvectors of a certain auxiliary integrable system. Commuting integrals of this auxiliary system are generated by the off-diagonal elements of the monodromy matrix of the BBS model. 
Then the eigenvectors of the auxiliary system serve as building blocks for the eigenvectors of the periodic BBS model. The multi-variable kernel which relates the respective eigenvectors can be presented as the product of single variable functions (SoV). Each of these functions satisfies the Baxter equation. In [1] we showed that the existence of non-trivial solutions of these equations is equivalent to the well known functional relations [2, 4, 5] for the transfer matrix of BBS model.

The goal of this paper is to use the explicit constructions of [1] for calculating matrix elements or form factors of local operators of the BBS model. Since the eigenvectors are given in terms of multiple summations, the main concern of our calculations is to perform these sums explicitly in order to get factorized expressions for the form factors and norms. For $N=2$ from the results of our first paper we calculate spin operator matrix elements of the generalized Ising model.

We hope that the methods developed in [1] and in this paper will allow to find analytical formulas for matrix elements of the CPM.

We would like to mention the paper [13], where matrix elements of local operators for the Toda chain are calculated using the eigenvectors of [12].

This paper is organized as follows: In Section 2 and 3 we recall the results of [1] for the right eigenfunctions of $B_{n}(\lambda)$, adding the analogous results for the respective left eigenfunctions, pointing out the differences. Then we calculate the overlap of the right and left eigenfunctions of $B_{n}(\lambda)$, i.e. the norms. Here the main result will be that the sum over intermediate quantum numbers can be performed explicitly. In Section 5 we concentrate on the case $N=2$ and calculate the overlap of the eigenvectors of the periodic transfer matrix $t(\lambda)=A_{n}(\lambda)+D_{n}(\lambda)$ for the homogeneous BBS model. At the end of Section 5 we also write a result for the matrix elements of the single particle operators $\mathbf{u}_{n}$ which still has multiple summations over discrete internal variables. In Section 6, using explicit solutions of the Baxter equation for the case of the homogeneous Ising model, we announce the factorized expression for the matrix elements of the one site spin operator where all internal summations have been performed. The details of this calculation will be given in a forthcoming paper [16]. These will prove a result conjectured by A. Bugrij and O. Lisovyy for the matrix elements of the spin operator in the Ising model on a finite lattice [14, 15. Finally, Section 7 summarizes our results and the Appendix explains our summation technique.

\section{L-operator formulation of the BBS-model}

We define the BBS-model as a quantum chain model in the $L$-operator vertex formulation (the relation to the formulation as a face model can be found in [1, 2]). To each site $k$ of the quantum chain we associate a cyclic $L$-operator [7, 4, 11] acting in a two-dimensional auxiliary space

$$
L_{k}(\lambda)=\left(\begin{array}{cc}
1+\lambda \varkappa_{k} \mathbf{v}_{k}, & \lambda \mathbf{u}_{k}^{-1}\left(a_{k}-b_{k} \mathbf{v}_{k}\right) \\
\mathbf{u}_{k}\left(c_{k}-d_{k} \mathbf{v}_{k}\right), & \lambda a_{k} c_{k}+\mathbf{v}_{k} b_{k} d_{k} / \varkappa_{k}
\end{array}\right), \quad k=1,2, \ldots, n .
$$


At each site $k$ there are ultra-local Weyl elements $\mathbf{u}_{k}$ and $\mathbf{v}_{k}$ obeying the commutation rules and normalization

$\mathbf{u}_{j} \mathbf{u}_{k}=\mathbf{u}_{k} \mathbf{u}_{j}, \quad \mathbf{v}_{j} \mathbf{v}_{k}=\mathbf{v}_{k} \mathbf{v}_{j}, \quad \mathbf{u}_{j} \mathbf{v}_{k}=\omega^{\delta_{j, k}} \mathbf{v}_{k} \mathbf{u}_{j}, \quad \omega=e^{2 \pi i / N}, \quad \mathbf{u}_{k}^{N}=\mathbf{v}_{k}^{N}=1$.

$\lambda$ is the spectral parameter. We have five parameters $\varkappa_{k}, a_{k}, b_{k}, c_{k}, d_{k}$ per site. At each site $k$ we define a $N$-dimensional linear space (quantum space) $\mathcal{V}_{k}$ with the basis $|\gamma\rangle_{k}, \gamma \in \mathbb{Z}_{N}$, the dual space $\mathcal{V}_{k}^{*}$ with the basis ${ }_{k}\langle\gamma|, \gamma \in \mathbb{Z}_{N}$, and the natural pairing ${ }_{k}\left\langle\gamma^{\prime} \mid \gamma\right\rangle_{k}=\delta_{\gamma^{\prime}, \gamma}$. In $\mathcal{V}_{k}$ the Weyl elements $\mathbf{u}_{k}$ and $\mathbf{v}_{k}$ act by the formulas:

$$
\mathbf{u}_{k}|\gamma\rangle_{k}=\omega^{\gamma}|\gamma\rangle_{k}, \quad \mathbf{v}_{k}|\gamma\rangle_{k}=|\gamma+1\rangle_{k}
$$

In $\mathcal{V}_{k}^{*}$ these Weyl elements act as follows:

$$
{ }_{k}\langle\gamma| \mathbf{u}_{k}={ }_{k}\langle\gamma| \omega^{\gamma}, \quad{ }_{k}\langle\gamma| \mathbf{v}_{k}={ }_{k}\langle\gamma-1| .
$$

The monodromy matrix for the quantum chain with $n$ sites is defined by

$$
T_{n}(\lambda)=L_{1}(\lambda) L_{2}(\lambda) \cdots L_{n}(\lambda)=\left(\begin{array}{cc}
A_{n}(\lambda) & B_{n}(\lambda) \\
C_{n}(\lambda) & D_{n}(\lambda)
\end{array}\right) .
$$

The transfer-matrix is defined to be the trace in the auxiliary space $\mathbf{t}_{n}(\lambda)=$ $\operatorname{tr} T_{n}(\lambda)=A_{n}(\lambda)+D_{n}(\lambda)$. This quantum chain is integrable because the $L$-operators (11) are intertwined by the twisted 6-vertex $R$-matrix at roots of unity. It leads to $\left[\mathbf{t}_{n}(\lambda), \mathbf{t}_{n}(\mu)\right]=0$ and so $\mathbf{t}_{n}(\lambda)$ is the generating function for the commuting set of non-local and non-hermitian Hamiltonians $\mathbf{H}_{0}, \ldots, \mathbf{H}_{n}$ of the model:

$$
\mathbf{t}_{n}(\lambda)=\mathbf{H}_{0}+\mathbf{H}_{1} \lambda+\cdots+\mathbf{H}_{n-1} \lambda^{n-1}+\mathbf{H}_{n} \lambda^{n} .
$$

From the intertwining relation, it follows that $B_{n}(\lambda)$ is the generating function for another commuting set of operators $\mathbf{h}_{1}, \ldots, \mathbf{h}_{n}$ :

$$
\left[B_{n}(\lambda), B_{n}(\mu)\right]=0, \quad B_{n}(\lambda)=\mathbf{h}_{1} \lambda+\mathbf{h}_{2} \lambda^{2}+\cdots+\mathbf{h}_{n} \lambda^{n} .
$$

Following Sklyanin [9], we shall first solve the eigenvalue problem for this last set of commuting operators, which turns out to be possible by a recursive procedure. After this, the periodic problem will be related to this auxiliary result by Baxter equations. After proper normalization by a Sklyanin's measure, the kernel of the $Q$-operator factorizes into a product of single variable functions (SoV).

\section{Eigenvectors of $B_{n}(\lambda)$}

\subsection{New basis vectors, one-site states}

In the root of unity case an important role is played by the cyclic function $w_{p}(\gamma)$ [17] which depends on the $\mathbb{Z}_{N}$-variable $\gamma$ and on a point $p=(x, y)$ on the Fermat curve $x^{N}+y^{N}=1$. We define $w_{p}(\gamma)$ by the difference equation

$$
\frac{w_{p}(\gamma)}{w_{p}(\gamma-1)}=\frac{y}{1-\omega^{\gamma} x}, \quad w_{p}(0)=1, \quad \gamma \in \mathbb{Z}_{N}
$$


The Fermat curve restriction guarantees the cyclic property: $w_{p}(\gamma+N)=w_{p}(\gamma)$. The function $w_{p}(\gamma)$ is a root of unity analog of the $q$-gamma function.

It is convenient to change the bases in the spaces $\mathcal{V}_{k}$ and $\mathcal{V}_{k}^{*}$. Instead of $|\gamma\rangle_{k}$ and ${ }_{k}\langle\gamma|, \gamma \in \mathbb{Z}_{N}$, we will use the vectors

$\left|\psi_{\rho}\right\rangle_{k}=\sum_{\gamma} w_{p_{k}^{\mathrm{R}}}(\gamma-\rho)|\gamma\rangle_{k}, \quad{ }_{k}\left\langle\psi_{\rho}\right|=\sum_{\gamma} \frac{1}{w_{p_{k}^{\mathrm{L}}}(\gamma-\rho-1)} k_{k}\langle\gamma|, \quad \rho \in \mathbb{Z}_{N}$.

The coordinates of the Fermat curve points $p_{k}^{\mathrm{L}}=\left(x_{k}^{\mathrm{L}}, y_{k}^{\mathrm{L}}\right)$ and $p_{k}^{\mathrm{R}}=\left(x_{k}^{\mathrm{R}}, y_{k}^{\mathrm{R}}\right)$ are defined as follows. Let us fix some value of $r_{k}$ to satisfy $r_{k}^{N}=a_{k}^{N}-b_{k}^{N}$. (We shall consider generic parameters such that $r_{k} \neq 0$.) Then

$$
x_{k}=x_{k}^{\mathrm{L}}=x_{k}^{\mathrm{R}}=\frac{r_{k}}{a_{k}}, \quad y_{k}=y_{k}^{\mathrm{R}}=\frac{b_{k}}{a_{k}}, \quad y_{k}^{\mathrm{L}}=\frac{b_{k}}{\omega a_{k}} .
$$

Observe that $y_{k}^{\mathrm{R}}=\omega y_{k}^{\mathrm{L}}$, while $x_{k}^{\mathrm{L}}=x_{k}^{\mathrm{R}}$. The vectors (44) are eigenvectors of the upper off-diagonal matrix element $\lambda \mathbf{u}_{k}^{-1}\left(a_{k}-b_{k} \mathbf{v}_{k}\right)$ of the operator $L_{k}$ :

$$
\begin{aligned}
& \lambda \mathbf{u}_{k}^{-1}\left(a_{k}-b_{k} \mathbf{v}_{k}\right)\left|\psi_{\rho}\right\rangle_{k}=\lambda r_{k} \omega^{-\rho}\left|\psi_{\rho}\right\rangle_{k}, \\
& { }_{k}\left\langle\psi_{\rho}\right| \lambda \mathbf{u}_{k}^{-1}\left(a_{k}-b_{k} \mathbf{v}_{k}\right)=\lambda r_{k} \omega^{-\rho}{ }_{k}\left\langle\psi_{\rho}\right| .
\end{aligned}
$$

The first equality has been proved in [1], the second relation follows very similarly. The action of the operator $\mathbf{v}_{k}$ is

$$
{ }_{k}\left\langle\psi_{\rho}\left|\mathbf{v}_{k}={ }_{k}\left\langle\psi_{\rho-1}\left|, \quad \mathbf{v}_{k}\right| \psi_{\rho}\right\rangle_{k}=\right| \psi_{\rho+1}\right\rangle_{k} .
$$

\subsection{Right and left eigenvectors of $B_{n}(\lambda)$ for general chain length $n$}

The eigenvectors of $B_{n}(\lambda)$ are labelled by the vector $\boldsymbol{\rho}_{n}=\left(\rho_{n, 0}, \ldots, \rho_{n, n-1}\right) \in\left(\mathbb{Z}_{N}\right)^{n}$. Let us further define:

$$
\tilde{\rho}_{n}=\sum_{k=0}^{n-1} \quad \rho_{n, k} ; \quad \boldsymbol{\rho}_{n}^{\prime}=\left(\rho_{n, 1}, \ldots, \rho_{n, n-1}\right) \in\left(\mathbb{Z}_{N}\right)^{n-1}
$$

and $\boldsymbol{\rho}_{n}^{ \pm k}$ denotes the vector $\boldsymbol{\rho}_{n}$ in which $\rho_{n, k}$ is replaced by $\rho_{n, k} \pm 1$, i.e.

$$
\boldsymbol{\rho}_{n}^{ \pm k}=\left(\rho_{n, 0}, \ldots, \rho_{n, k} \pm 1, \ldots, \rho_{n, n-1}\right), \quad k=0,1, \ldots, n-1 .
$$

The following formulas give an iterative procedure to obtain the eigenvectors of $B_{n}(\lambda)$ from eigenvectors of $B_{n-1}(\lambda)$ and single site vectors defined by (4).

The vector

$$
\left|\Psi_{\boldsymbol{\rho}_{n}}\right\rangle=\sum_{\substack{\rho_{n-1} \in\left(\mathbb{Z}_{N}\right)^{n-1} \\ \rho_{n} \in \mathbb{Z}_{N}}} Q^{R}\left(\boldsymbol{\rho}_{n-1}, \rho_{n} \mid \boldsymbol{\rho}_{n}\right)\left|\Psi_{\boldsymbol{\rho}_{n-1}}\right\rangle \otimes\left|\psi_{\rho_{n}}\right\rangle_{n}
$$

where

$$
\begin{aligned}
Q^{R}\left(\boldsymbol{\rho}_{n-1}, \rho_{n} \mid \boldsymbol{\rho}_{n}\right)=\frac{\omega^{\left(\tilde{\rho}_{n}-\tilde{\rho}_{n-1}\right)\left(\rho_{n}-\rho_{n, 0}\right)}}{w_{p_{n, 0}}\left(\rho_{n, 0}-\rho_{n-1,0}-1\right) w_{\tilde{p}_{n}}\left(\tilde{\rho}_{n}-\rho_{n}-1\right)} \times \\
\times \frac{\prod_{l=1}^{n-2} \prod_{k=1}^{n-1} w_{p_{n-1, l}^{n, k}}\left(\rho_{n-1, l}-\rho_{n, k}\right)}{\prod_{\substack{n, l=1 \\
j \neq l}}^{n-2} w_{p_{n-1, j}^{n-1, l}}\left(\rho_{n-1, j}-\rho_{n-1, l}\right)} \prod_{l=1}^{n-2} \frac{w_{p_{n-1, l}}\left(-\rho_{n-1, l}\right)}{w_{\tilde{p}_{n-1, l}}\left(\rho_{n-1, l}\right)}
\end{aligned}
$$

is right eigenvector of $B_{n}(\lambda)$ : 
Form-factors in the BBS model I: Norms and matrix elements

$$
B_{n}(\lambda)\left|\Psi_{\rho_{n}}\right\rangle=\lambda r_{n, 0} \omega^{-\rho_{n, 0}} \prod_{k=1}^{n-1}\left(\lambda+r_{n, k} \omega^{-\rho_{n, k}}\right)\left|\Psi_{\rho_{n}}\right\rangle
$$

if $\left|\Psi_{\boldsymbol{\rho}_{n-1}}\right\rangle$ is right eigenvector of $B_{n-1}(\lambda)$. The Fermat curve points $\tilde{p}_{n}, p_{n, l}, \tilde{p}_{n, l}, p_{n^{\prime}, l}^{n, k}$ and $r_{n, k}$ entering (8), (9) are related to the parameters of the model $a_{s}, b_{s}, c_{s}, d_{s}, \varkappa_{s}$ by equations (54)-(61) of [1], e.g. $\tilde{p}_{n, l}=\left(\tilde{x}_{n, l}, \tilde{y}_{n, l}\right), \quad \tilde{x}_{n, l}=d_{n} /\left(\varkappa_{n} c_{n} r_{n, l}\right), \quad x_{n-1, l}^{n, k}=r_{n, k} / r_{n-1, l}$. For the more involved determination of the $r_{n, l}$, see Section 2.3 of [1].

Analogously, the vector

where

$$
\left\langle\Psi_{\boldsymbol{\rho}_{n}}\right|=\sum_{\substack{\boldsymbol{\rho}_{n-1} \in\left(\mathbb{Z}_{N}\right)^{n-1} \\ \rho_{n} \in \mathbb{Z}_{N}}} Q^{\mathrm{L}}\left(\boldsymbol{\rho}_{n-1}, \rho_{n} \mid \boldsymbol{\rho}_{n}\right)\left\langle\Psi_{\boldsymbol{\rho}_{n-1}}\right| \otimes_{n}\left\langle\psi_{\rho_{n}}\right|
$$

$$
\begin{aligned}
Q^{\mathrm{L}}\left(\boldsymbol{\rho}_{n-1}, \rho_{n} \mid \boldsymbol{\rho}_{n}\right)= & \omega^{-\left(\tilde{\rho}_{n}-\tilde{\rho}_{n-1}\right)\left(\rho_{n}-\rho_{n, 0}\right)+\tilde{\rho}_{n-1}-\rho_{n-1,0}} \prod_{\substack{j, l=1 \\
j \neq l}}^{n-2} w_{p_{n-1, j}^{n-1, l}}\left(\rho_{n-1, j}-\rho_{n-1, l}-1\right) \times \\
& \times \frac{w_{p_{n, 0}}\left(\rho_{n, 0}-\rho_{n-1,0}\right) w_{\tilde{p}_{n}}\left(\tilde{\rho}_{n}-\rho_{n}\right)}{\prod_{l=1}^{n-2} \prod_{k=1}^{n-1} w_{p_{n-1, l}^{n, k}}\left(\rho_{n-1, l}-\rho_{n, k}-1\right)} \prod_{l=1}^{n-2} \frac{w_{\tilde{p}_{n-1, l}}\left(\rho_{n-1, l}\right)}{w_{p_{n-1, l}}\left(-\rho_{n-1, l}\right)},
\end{aligned}
$$

is left eigenvector of $B_{n}(\lambda)$ :

$$
\left\langle\Psi_{\boldsymbol{\rho}_{n}}\right| B_{n}(\lambda)=\lambda r_{n, 0} \omega^{-\rho_{n, 0}} \prod_{k=1}^{n-1}\left(\lambda+r_{n, k} \omega^{-\rho_{n, k}}\right)\left\langle\Psi_{\boldsymbol{\rho}_{n}}\right|
$$

if $\left\langle\Psi_{\boldsymbol{\rho}_{n-1}}\right|$ is left eigenvector of $B_{n-1}(\lambda)$. The definition of $Q^{\mathrm{L}}\left(\boldsymbol{\rho}_{n-1}, \rho_{n} \mid \boldsymbol{\rho}_{n}\right)$ uses the same Fermat curve points $\tilde{p}_{n}, p_{n, l}, \tilde{p}_{n, l}, p_{n^{\prime}, l}^{n, k}$ as were used for the right eigenvectors. The proof of (11) by induction is lengthy, but analogous to the proof of (8) given in [1].

At $\lambda$ equal to one of the $n-1$ zeros $\lambda_{n, k}$ of the eigenvalue polynomial of $B_{n}(\lambda)$

$$
\lambda_{n, k}=-r_{n, k} \omega^{-\rho_{n, k}}, \quad k=1, \ldots, n-1,
$$

the operators $A_{n}\left(\lambda_{n, k}\right)$ and $D_{n}\left(\lambda_{n, k}\right)$ act as shift operators for the $k$-th component of the vector $\boldsymbol{\rho}_{n}, D_{n}\left(\lambda_{n, k}\right)$ shifting in addition also the zeroth component:

$$
\begin{aligned}
A_{n}\left(\lambda_{n, k}\right)\left|\Psi_{\boldsymbol{\rho}_{n}}\right\rangle & =\varphi_{k}\left(\boldsymbol{\rho}_{n}^{\prime}\right)\left|\Psi_{\boldsymbol{\rho}_{n}^{+k}}\right\rangle, & & \left\langle\Psi_{\boldsymbol{\rho}_{n}}\right| A_{n}\left(\lambda_{n, k}\right)=\omega^{-1} \varphi_{k}\left(\boldsymbol{\rho}_{n}^{\prime-k}\right)\left\langle\Psi_{\boldsymbol{\rho}_{n}^{-k}}\right|, \\
D_{n}\left(\lambda_{n, k}\right)\left|\Psi_{\boldsymbol{\rho}_{n}}\right\rangle & =\tilde{\varphi}_{k}\left(\boldsymbol{\rho}_{n}^{\prime}\right)\left|\Psi_{\boldsymbol{\rho}_{n}^{+0,-k}}\right\rangle, & & \left\langle\Psi_{\boldsymbol{\rho}_{n}}\right| D_{n}\left(\lambda_{n, k}\right)=\omega \tilde{\varphi}_{k}\left(\boldsymbol{\rho}_{n}^{\prime+k}\right)\left\langle\Psi_{\boldsymbol{\rho}_{n}^{-0,+k}}\right|
\end{aligned}
$$

where

$$
\begin{aligned}
& \varphi_{k}\left(\boldsymbol{\rho}_{n}^{\prime}\right)=-\frac{\tilde{r}_{n-1}}{r_{n}} \omega^{-\tilde{\rho}_{n}+\rho_{n, 0}} F_{n}\left(\lambda_{n, k} / \omega\right) \prod_{s=1}^{n-2} y_{n-1, s}^{n, k}, \\
& \tilde{\varphi}_{k}\left(\boldsymbol{\rho}_{n}^{\prime}\right)=-\frac{r_{n}}{\tilde{r}_{n-1}} \frac{\omega^{\tilde{\rho}_{n}-\rho_{n, 0}-1}}{\prod_{s=1}^{n-2} y_{n-1, s}^{n, k}} \prod_{m=1}^{n-1} F_{m}\left(\lambda_{n, k}\right)
\end{aligned}
$$

and

$$
F_{m}(\lambda)=\left(b_{m}+\omega a_{m} \varkappa_{m} \lambda\right)\left(\lambda c_{m}+d_{m} / \varkappa_{m}\right) .
$$

The operator $\mathbf{V}_{n}=\mathbf{v}_{1} \mathbf{v}_{2} \ldots \mathbf{v}_{n}$, which defines the term of highest degree in $\lambda$ in $A_{n}(\lambda)$ and the free term in $D_{n}(\lambda)$, shifts the zeroth index of vector $\boldsymbol{\rho}_{n}$ :

$$
\mathbf{V}_{n}\left|\Psi_{\boldsymbol{\rho}_{n}}\right\rangle=\left|\Psi_{\boldsymbol{\rho}_{n}^{+0}}\right\rangle, \quad\left\langle\Psi_{\boldsymbol{\rho}_{n}}\right| \mathbf{V}_{n}=\left\langle\Psi_{\boldsymbol{\rho}_{n}^{-0}}\right|
$$


Using interpolation polynomials and formulas (13), (14) and (17), one can construct how $A_{n}(\lambda)$ and $D_{n}(\lambda)$ act on the left eigenvectors of $B_{n}(\lambda)$, analogously to what was done in equations (66)-(68) of [1] for the right eigenvectors.

\subsection{The norms of the eigenvectors of $B_{n}(\lambda)$}

The pairing ${ }_{k}\left\langle\gamma^{\prime} \mid \gamma\right\rangle_{k}=\delta_{\gamma^{\prime}, \gamma}$ implies (we use $y_{k} \equiv y_{k}^{R}=\omega y_{k}^{L}$ ):

$$
{ }_{k}\left\langle\psi_{\rho^{\prime}} \mid \psi_{\rho}\right\rangle_{k}=\delta_{\rho^{\prime}, \rho} \frac{N}{\omega}\left(\frac{x_{k}}{y_{k}}\right)^{N-1} .
$$

Proof. From (44) we get directly

$$
{ }_{k}\left\langle\psi_{\rho^{\prime}} \mid \psi_{\rho}\right\rangle_{k}=\sum_{\gamma \in \mathbb{Z}_{N}} \frac{w_{p_{k}^{\mathrm{L}}}(\gamma) \omega^{\gamma}}{w_{p_{k}^{\mathrm{L}}}\left(\gamma+\rho-\rho^{\prime}-1\right)}
$$

since $w_{p_{k}^{\mathrm{R}}}(\gamma)=w_{p_{k}^{\mathrm{L}}}(\gamma) \omega^{\gamma}$. States for $\rho^{\prime} \neq \rho$ belong to different eigenvalues in ([5), (6) so they are "orthogonal" (have vanishing pairing). We then use (3) and

$$
\begin{aligned}
& \frac{y^{N}}{1-\omega^{\gamma} x}=\sum_{\gamma^{\prime}=0}^{N-1} \omega^{\gamma \gamma^{\prime}} x^{\gamma^{\prime}}, \quad \sum_{\gamma \in \mathbb{Z}_{N}} \frac{1}{1-\omega^{\gamma} x}=\frac{N}{y^{N}}, \\
& \text { and for } 1 \leq \alpha \leq N: \quad \sum_{\gamma \in \mathbb{Z}_{N}} \frac{\omega^{\alpha \gamma}}{1-\omega^{\gamma} x}=N \frac{x^{N-\alpha}}{y^{N}} .
\end{aligned}
$$

Next, we calculate the left-right overlap of the eigenstates of $B_{n}(\lambda)$ for general $n$. We shall consider only the generic case of parameters such that all the eigenvalues of $B_{n}(\lambda)$ are different (in particular, this is important for the action of $A_{n}(\lambda)$ and $D_{n}(\lambda)$

on the eigenvectors of $\left.B_{n}(\lambda)\right)$. Therefore $\left\langle\Psi_{\boldsymbol{\rho}_{n}^{L}} \mid \Psi_{\boldsymbol{\rho}_{n}^{R}}\right\rangle=0$ if $\boldsymbol{\rho}_{n}^{L} \neq \boldsymbol{\rho}_{n}^{R}$. The following theorem gives the value of $\left\langle\Psi_{\boldsymbol{\rho}_{n}^{L}} \mid \Psi_{\boldsymbol{\rho}_{n}^{R}}\right\rangle$ ("norm") for $\boldsymbol{\rho}_{n}^{L}=\boldsymbol{\rho}_{n}^{R}=\boldsymbol{\rho}_{n}$ :

Theorem 1 The norms $\left\langle\Psi_{\boldsymbol{\rho}_{n}} \mid \Psi_{\boldsymbol{\rho}_{n}}\right\rangle$ are independent of the phase $\rho_{n, 0}$ and their dependence on $\boldsymbol{\rho}_{n}^{\prime}=\left(\rho_{n, 1}, \ldots, \rho_{n, n-1}\right)$ is given explicitly as

$$
\left\langle\Psi_{\boldsymbol{\rho}_{n}} \mid \Psi_{\boldsymbol{\rho}_{n}}\right\rangle=\frac{C_{n}}{\prod_{l<m}\left(\lambda_{n, l}-\lambda_{n, m}\right)}=\frac{C_{n}}{\prod_{l<m}\left(r_{n, m} \omega^{-\rho_{n, m}}-r_{n, l} \omega^{\left.-\rho_{n, l}\right)}\right.},
$$

where the normalizing factor $C_{n}$ is independent of $\boldsymbol{\rho}_{n}$ and it is defined recursively by

$C_{n}=C_{n-1} \mathcal{N}_{n} N^{n-2} \frac{\prod_{k<k^{\prime}}\left(r_{n, k}^{N}-r_{n, k^{\prime}}^{N}\right) \prod_{l<l^{\prime}}\left(r_{n-1, l^{\prime}}^{N}-r_{n-1, l}^{N}\right)}{\prod_{k, l}\left(r_{n, k}^{N}-r_{n-1, l}^{N}\right)} \prod_{l=1}^{n-2} r_{n-1, l}^{N+1}$,

$C_{1}$ and $C_{2}$ are given by

$$
C_{1}=\frac{N}{\omega}\left(\frac{x_{1}}{y_{1}}\right)^{N-1}, \quad C_{2}=C_{1} \frac{N^{3}}{\omega}\left(\frac{x_{2}}{y_{2} \tilde{y}_{2} y_{2,0}}\right)^{N-1}
$$

and

$$
\mathcal{N}_{n}=\frac{N^{3}}{\omega}\left(\frac{x_{n}}{y_{n} y_{n, 0} \tilde{y}_{n}}\right)^{N-1}\left(\prod_{l=1}^{n-2} \frac{\prod_{k=1}^{n-1} y_{n-1, l}^{n, k}}{\prod_{j, j \neq l}^{n-2} y_{n-1, l}^{n-1, j}}\right)
$$


Proof. We shall give the proof by establishing an explicit recursion $C_{n-1} \rightarrow C_{n}$. For $n=2$ there is only a single zero $r_{2,1} \omega^{-\rho_{2,1}}$ and the denominator in (20) is unity. Similarly, for $n=1$ this denominator is unity too as we see from (18).

From (7) and (10) we get

$$
\left\langle\Psi_{\boldsymbol{\rho}_{n}} \mid \Psi_{\boldsymbol{\rho}_{n}}\right\rangle=\sum_{\substack{\boldsymbol{\rho}_{n-1} \in\left(\mathbb{Z}_{N}\right)^{n-1} \\ \rho_{n} \in \mathbb{Z}_{N}}} Q^{\mathrm{L}}\left(\boldsymbol{\rho}_{n-1}, \rho_{n} \mid \boldsymbol{\rho}_{n}\right) Q^{\mathrm{R}}\left(\boldsymbol{\rho}_{n-1}, \rho_{n} \mid \boldsymbol{\rho}_{n}\right)\left\langle\Psi_{\boldsymbol{\rho}_{n-1}} \mid \Psi_{\boldsymbol{\rho}_{n-1}}\right\rangle{ }_{n}\left\langle\psi_{\rho_{n}} \mid \psi_{\rho_{n}}\right\rangle_{n} .
$$

Inserting here the explicit expressions (111) for $Q^{\mathrm{L}}$, and (8) for $Q^{\mathrm{R}}$, using (18), and performing the summations over $\rho_{n-1,0}$ and $\rho_{n}$ by (19) we get:

$$
\begin{aligned}
& \left\langle\Psi_{\boldsymbol{\rho}_{n}} \mid \Psi_{\boldsymbol{\rho}_{n}}\right\rangle=\mathcal{N}_{n} \sum_{\boldsymbol{\rho}_{n-1}^{\prime} \in\left(\mathbb{Z}_{N}\right)^{n-2}} \omega^{\rho_{n-1,1}+\ldots+\rho_{n-1, n-2}} \times \\
& \quad \times \prod_{l=1}^{n-2}\left(r_{n-1, l} \omega^{-\rho_{n-1, l}}\right)^{2} \frac{\prod_{j, j \neq l}^{n-2}\left(r_{n-1, j} \omega^{-\rho_{n-1, j}}-r_{n-1, l} \omega^{-\rho_{n-1, l}}\right)}{\prod_{k=1}^{n-1}\left(r_{n-1, l} \omega^{-\rho_{n-1, l}}-r_{n, k} \omega^{-\rho_{n, k}}\right)}\left\langle\Psi_{\boldsymbol{\rho}_{n-1}} \mid \Psi_{\boldsymbol{\rho}_{n-1}}\right\rangle,
\end{aligned}
$$

where we combined several phase-independent factors into the quantity (22). The main issue now is to perform the summations over the phases $\boldsymbol{\rho}_{n-1}^{\prime}$ explicitly. In order to avoid too many indices, let us define

$$
\begin{aligned}
& \mathbf{a}^{(n)}=\left(a_{1}, \ldots, a_{n-2}\right)=\left(r_{n-1,1} \omega^{-\rho_{n-1,1}}, \ldots, r_{n-1, n-2} \omega^{-\rho_{n-1, n-2}}\right) ; \\
& \mathbf{b}^{(n)}=\left(b_{1}, \ldots, b_{n-1}\right)=\left(-\lambda_{n, 1}, \ldots,-\lambda_{n, n-1}\right)=\left(r_{n, 1} \omega^{-\rho_{n, 1}}, \ldots, r_{n, n-1} \omega^{-\rho_{n, n-1}}\right) .
\end{aligned}
$$

Then using the induction assumption that the formula for the norms is given by (20), the relation (24) reduces to

$$
\begin{aligned}
\frac{C_{n}}{\prod_{l<m}\left(b_{m}-b_{l}\right)} & =\mathcal{N}_{n} \sum_{\rho_{n-1,1}, \ldots, \rho_{n-1, n-2}} \prod_{l=1}^{n-2} r_{n-1, l} a_{l} \frac{\prod_{j \neq l}^{n-2}\left(a_{j}-a_{l}\right)}{\prod_{k=1}^{n-1}\left(a_{l}-b_{k}\right)} \frac{C_{n-1}}{\prod_{m=l+1}^{n-2}\left(a_{m}-a_{l}\right)} \\
& =C_{n-1} \mathcal{N}_{n} \tilde{r}_{n-1}^{\prime} S_{n}\left(\mathbf{a}^{(n)}, \mathbf{b}^{(n)}\right),
\end{aligned}
$$

where we define $\tilde{r}_{n-1}^{\prime}=r_{n-1,1} \ldots r_{n-1, n-2}$ and

$$
S_{n}\left(\mathbf{a}^{(n)}, \mathbf{b}^{(n)}\right)=\sum_{\rho_{n-1,1}, \ldots, \rho_{n-1, n-2} \in\left(\mathbb{Z}_{N}\right)^{n-2}} \prod_{l=1}^{n-2}\left(a_{l} \frac{\prod_{j=l+1}^{n-2}\left(a_{l}-a_{j}\right)}{\prod_{k=1}^{n-1}\left(a_{l}-b_{k}\right)}\right) .
$$

In the Appendix, we prove the following identity

$$
S_{n}\left(\mathbf{a}^{(n)}, \mathbf{b}^{(n)}\right)=N^{n-2}\left(\prod_{l=1}^{n-2} a_{l}^{N}\right) \prod_{k<k^{\prime}}^{n-1} \frac{b_{k}^{N}-b_{k^{\prime}}^{N}}{b_{k}-b_{k^{\prime}}} \frac{\prod_{l<l^{\prime}}^{n-2}\left(a_{l}^{N}-a_{l^{\prime}}^{N}\right)}{\prod_{l=1}^{n-1} \prod_{l^{\prime}=1}^{n-2}\left(b_{l}^{N}-a_{l^{\prime}}^{N}\right)} .
$$

The $\left(\mathbb{Z}_{N}\right)^{n-2}$-summation in (27) runs over the discrete phases of the variables $\mathbf{a}^{(n)}$. From the $\mathbb{Z}_{N}$-symmetry the result of this summation (28) can depend only on the $N$-th powers of these variables. Substituting (28) into (26), and using the notation (25), we get the recursion (21). 


\subsection{Action of $\mathbf{u}_{n}$ on $B_{n}(\lambda)$-eigenvectors}

Now we shall calculate an explicit expression for the action of $\mathbf{u}_{n}$ on the $B_{n}(\lambda)$ eigenvectors $\left|\Psi_{\boldsymbol{\rho}_{n}}\right\rangle$. From the relation

$$
\mathbf{u}_{n}^{-1}\left(a_{n}-b_{n} \mathbf{v}_{n}\right)\left|\psi_{\rho_{n}}\right\rangle_{n}=r_{n} \omega^{-\rho_{n}}\left|\psi_{\rho_{n}}\right\rangle_{n}
$$

taking into account $a_{n} / r_{n}=1 / x_{n}, b_{n} / r_{n}=y_{n} / x_{n}$, we get the formula for the action of $\mathbf{u}_{n}$ on one-site eigenvectors:

$$
\mathbf{u}_{n}\left|\psi_{\rho_{n}}\right\rangle_{n}=\omega^{\rho_{n}}\left(\frac{1}{x_{n}}\left|\psi_{\rho_{n}}\right\rangle_{n}-\frac{y_{n}}{x_{n}}\left|\psi_{\rho_{n}+1}\right\rangle_{n}\right) .
$$

We use this formula to obtain the action of $\mathbf{u}_{n}$ on general eigenvectors of $B_{n}(\lambda)$, which, in particular, will show that most matrix elements of $\mathbf{u}_{n}$ between eigenvectors of $B_{n}(\lambda)$ vanish:

Theorem 2 The action of $\mathbf{u}_{n}$ on eigenvectors of $B_{n}(\lambda)$ is given by

$$
\begin{aligned}
& \mathbf{u}_{n}\left|\Psi_{\boldsymbol{\rho}_{n}}\right\rangle=\omega^{\tilde{\rho}_{n}} \frac{\tilde{x}_{n}}{x_{n}}\left|\Psi_{\boldsymbol{\rho}_{n}}\right\rangle-\omega^{\rho_{n, 0}} \frac{y_{n, 0} y_{n}}{x_{n}}\left|\Psi_{\boldsymbol{\rho}_{n}^{+0}}\right\rangle+ \\
& +\sum_{k=1}^{n-1} \omega^{\rho_{n, 0}-\tilde{\rho}_{n}^{\prime}}\left(\frac{\tilde{y}_{n} \tilde{r}_{n}}{\omega^{-\rho_{n, k}} r_{n, k} r_{n, 0} x_{n}}-\frac{x_{n, 0} \tilde{r}_{n-1} y_{n}}{\omega r_{n-1,0} x_{n}}\right) \frac{\prod_{l=1}^{n-2} y_{n-1, l}^{n, k}}{\prod_{s \neq k}\left(-r_{n, k} \omega^{-\rho_{n, k}}+r_{n, s} \omega^{-\rho_{n, s}}\right)}\left|\Psi_{\boldsymbol{\rho}_{n}^{+k}}\right\rangle .
\end{aligned}
$$

Proof. To prove (30) we use (7), (29) and rewrite (30) as a relation for $Q^{\mathrm{R}}\left(\boldsymbol{\rho}_{n-1}, \rho_{n} \mid \boldsymbol{\rho}_{n}\right)$. The terms of the resulting relation can be separated into two groups, giving two relations which can be proved independently:

$\omega^{\rho_{n}}=\omega^{\tilde{\rho}_{n}} \tilde{x}_{n}+\sum_{k=1}^{n-1} \frac{\omega^{\rho_{n, 0}-\tilde{\rho}_{n}^{\prime}} \tilde{y}_{n} \tilde{r}_{n}}{r_{n, k} \omega^{-\rho_{n, k}} r_{n, 0}} \frac{\prod_{l=1}^{n-2} y_{n-1, l}^{n, k}}{\prod_{s \neq k}\left(-r_{n, k} \omega^{-\rho_{n, k}}+r_{n, s} \omega^{-\rho_{n, s}}\right)} \frac{Q^{\mathrm{R}}\left(\boldsymbol{\rho}_{n-1}, \rho_{n} \mid \boldsymbol{\rho}_{n}^{+k}\right)}{Q^{\mathrm{R}}\left(\boldsymbol{\rho}_{n-1}, \rho_{n} \mid \boldsymbol{\rho}_{n}\right)}$

and

$$
\begin{aligned}
& \omega^{\rho_{n}-1} \frac{Q^{\mathrm{R}}\left(\boldsymbol{\rho}_{n-1}, \rho_{n}-1 \mid \boldsymbol{\rho}_{n}\right)}{Q^{\mathrm{R}}\left(\boldsymbol{\rho}_{n-1}, \rho_{n} \mid \boldsymbol{\rho}_{n}\right)}=\omega^{\rho_{n, 0}} y_{n, 0} \frac{Q^{\mathrm{R}}\left(\boldsymbol{\rho}_{n-1}, \rho_{n} \mid \boldsymbol{\rho}_{n}^{+0}\right)}{Q^{\mathrm{R}}\left(\boldsymbol{\rho}_{n-1}, \rho_{n} \mid \boldsymbol{\rho}_{n}\right)} \\
& +\sum_{k=1}^{n-1} \omega^{\rho_{n, 0}-\tilde{\rho}_{n}^{\prime}-1} \frac{x_{n, 0} \tilde{r}_{n-1}}{r_{n-1,0}} \frac{\prod_{l=1}^{n-2} y_{n-1, l}^{n, k}}{\prod_{s \neq k}\left(-r_{n, k} \omega^{-\rho_{n, k}}+r_{n, s} \omega^{-\rho_{n, s}}\right)} \frac{Q^{\mathrm{R}}\left(\boldsymbol{\rho}_{n-1}, \rho_{n} \mid \boldsymbol{\rho}_{n}^{+k}\right)}{Q^{\mathrm{R}}\left(\boldsymbol{\rho}_{n-1}, \rho_{n} \mid \boldsymbol{\rho}_{n}\right)} .
\end{aligned}
$$

In order to verify both (31) and (32) we start evaluating the ratios of the $Q^{R}$ using the explicit formula (8), e.g.

$\frac{Q^{\mathrm{R}}\left(\boldsymbol{\rho}_{n-1}, \rho_{n} \mid \boldsymbol{\rho}_{n}^{+k}\right)}{Q^{\mathrm{R}}\left(\boldsymbol{\rho}_{n-1}, \rho_{n} \mid \boldsymbol{\rho}_{n}\right)}=\omega^{\rho_{n}-\rho_{n, 0}} \frac{1-\omega^{\tilde{\rho}_{n}-\rho_{n}} \tilde{x}_{n}}{\tilde{y}_{n}} \prod_{l=1}^{n-2} \frac{r_{n-1, l} \omega^{-\rho_{n-1, l}}-r_{n, k} \omega^{-\rho_{n, k}}}{r_{n-1, l} \omega^{-\rho_{n-1, l}} y_{n-1, l}^{n, k}}$

since in (33) the shift $\boldsymbol{\rho}_{n}^{+k}$ affects only three terms in (81) and we use (3) and $x_{n-1, l}^{n, k}=r_{n, k} / r_{n-1, l}$. For (31), inserting (33), and after some cancellations, collecting the $k$-dependent terms, the sum over $k$ can be performed using the identity

$$
\sum_{k=1}^{n-1} \frac{\prod_{l=1}^{n-2}\left(\xi_{k}-\zeta_{l}\right)}{\xi_{k} \prod_{s=1, s \neq k}^{n-1}\left(\xi_{k}-\xi_{s}\right)}=\frac{\prod_{l=1}^{n-2} \zeta_{l}}{\prod_{k=1}^{n-1} \xi_{k}}, \quad \xi_{k}=-r_{n, k} \omega^{-\rho_{n, k}}, \quad \zeta_{l}=-r_{n-1, l} \omega^{-\rho_{n-1, l}} .
$$


For verifying (32) we have to calculate the other shifts of $Q^{R}$ too. This time, with the same $\xi_{k}$ and $\zeta_{l}$ as before, we perform the sum over $k$ using the identity

$$
\sum_{k=1}^{n-1} \frac{\prod_{l=1}^{n-2}\left(\xi_{k}-\zeta_{l}\right)}{\prod_{s=1, s \neq k}^{n-1}\left(\xi_{k}-\xi_{s}\right)}=1
$$

\section{Periodic model. Baxter equation}

To treat the periodic model it is convenient to make a Fourier transform over $\rho_{n, 0}$ of the eigenvectors of $B_{n}(\lambda)$. This yields a basis of eigenvectors of the operator $\mathbf{V}_{n}$ of (17):

$$
\begin{array}{ll}
\left\langle\tilde{\Psi}_{\rho, \boldsymbol{\rho}_{n}^{\prime}}\right|=\sum_{\rho_{n, 0} \in \mathbb{Z}_{N}} \omega^{\rho \cdot \rho_{n, 0}}\left\langle\Psi_{\boldsymbol{\rho}_{n}}\right|, & \left|\tilde{\Psi}_{\rho, \boldsymbol{\rho}_{n}^{\prime}}\right\rangle=\sum_{\rho_{n, 0} \in \mathbb{Z}_{N}} \omega^{-\rho \cdot \rho_{n, 0}}\left|\Psi_{\boldsymbol{\rho}_{n}}\right\rangle, \\
\left\langle\tilde{\Psi}_{\rho, \boldsymbol{\rho}_{n}^{\prime}}\left|\mathbf{V}_{n}=\omega^{\rho}\left\langle\tilde{\Psi}_{\rho, \boldsymbol{\rho}_{n}^{\prime}}\left|, \quad \mathbf{V}_{n}\right| \tilde{\Psi}_{\rho, \boldsymbol{\rho}_{n}^{\prime}}\right\rangle=\omega^{\rho}\right| \tilde{\Psi}_{\rho, \boldsymbol{\rho}_{n}^{\prime}}\right\rangle .
\end{array}
$$

$\mathbf{V}_{n}$ is the total $\mathbb{Z}_{N}$-spin rotation operator and $\rho \in \mathbb{Z}_{N}$ is the corresponding total charge.

Let $\left\langle\Phi_{\rho, \mathbf{E}}\right|$ and $\left|\Phi_{\rho, \mathbf{E}}\right\rangle$ be left and right eigenvectors of $\mathbf{t}_{n}(\lambda)$ with eigenvalue

$$
t_{n}(\lambda \mid \rho, \mathbf{E})=E_{0}+E_{1} \lambda+\cdots+E_{n-1} \lambda^{n-1}+E_{n} \lambda^{n},
$$

where $\mathbf{E}=\left\{E_{1}, \ldots, E_{n-1}\right\}$ and the values of $E_{0}$ and $E_{n}$ are

$$
E_{0}=1+\omega^{\rho} \prod_{m=1}^{n} \frac{b_{m} d_{m}}{\varkappa_{m}}, \quad E_{n}=\prod_{m=1}^{n} a_{m} c_{m}+\omega^{\rho} \prod_{m=1}^{n} \varkappa_{m} .
$$

Following the general procedure of the SoV method, we express these eigenvectors as

$$
\begin{gathered}
\left\langle\Phi_{\rho, \mathbf{E}}\left|=\sum_{\boldsymbol{\rho}_{n}^{\prime}} Q^{\mathrm{L}}\left(\boldsymbol{\rho}_{n}^{\prime} \mid \rho, \mathbf{E}\right)\left\langle\tilde{\Psi}_{\rho, \boldsymbol{\rho}_{n}^{\prime}}|, \quad| \Phi_{\rho, \mathbf{E}}\right\rangle=\sum_{\boldsymbol{\rho}_{n}^{\prime}} Q^{\mathrm{R}}\left(\boldsymbol{\rho}_{n}^{\prime} \mid \rho, \mathbf{E}\right)\right| \tilde{\Psi}_{\rho, \boldsymbol{\rho}_{n}^{\prime}}\right\rangle, \\
\text { wheret } Q^{\mathrm{L}}\left(\boldsymbol{\rho}_{n}^{\prime} \mid \rho, \mathbf{E}\right)=\prod_{k=1}^{n-1} Q_{k}^{\mathrm{L}}\left(\rho_{n, k}\right) \prod_{\substack{s, s^{\prime}=1 \\
s \neq s^{\prime}}}^{n-1} w_{p_{n, s}^{n, s^{\prime}}}\left(\rho_{n, s}-\rho_{n, s^{\prime}}-1\right), \\
Q^{\mathrm{R}}\left(\boldsymbol{\rho}_{n}^{\prime} \mid \rho, \mathbf{E}\right)=\frac{\prod_{k=1}^{n-1} Q_{k}^{\mathrm{R}}\left(\rho_{n, k}\right)}{\prod_{\substack{n, s^{\prime}=1 \\
s \neq s^{\prime}}}^{n-1} w_{p_{n, s}^{n, s^{\prime}}}\left(\rho_{n, s}-\rho_{n, s^{\prime}}\right)} .
\end{gathered}
$$

The products of $\mathbb{Z}_{N^{-}}$cyclic functions $w_{p_{n, s}^{n, s^{\prime}}}\left(\rho_{n, s}-\rho_{n, s^{\prime}}\right)$ in (39) and (40) are Sklyanin's measure which makes the rest of the kernels $Q^{\mathrm{L}}\left(\boldsymbol{\rho}_{n}^{\prime} \mid \rho, \mathbf{E}\right)$ and $Q^{\mathrm{R}}\left(\boldsymbol{\rho}_{n}^{\prime} \mid \rho, \mathbf{E}\right)$ factorizable into products of single variable functions (SoV). The Baxter equations for these functions of separated variables are

$$
\begin{aligned}
& t_{n}\left(\lambda_{n, k} \mid \rho, \mathbf{E}\right) Q_{k}^{\mathrm{L}}\left(\rho_{n, k}\right)=\omega^{n-1} \Delta_{k}^{-}\left(\lambda_{n, k}\right) Q_{k}^{\mathrm{L}}\left(\rho_{n, k}+1\right)+\omega^{1-n} \Delta_{k}^{+}\left(\omega \lambda_{n, k}\right) Q_{k}^{\mathrm{L}}\left(\rho_{n, k}-1\right), \\
& t_{n}\left(\lambda_{n, k} \mid \rho, \mathbf{E}\right) Q_{k}^{\mathrm{R}}\left(\rho_{n, k}\right)=\Delta_{k}^{+}\left(\lambda_{n, k}\right) Q_{k}^{\mathrm{R}}\left(\rho_{n, k}+1\right)+\Delta_{k}^{-}\left(\omega \lambda_{n, k}\right) Q_{k}^{\mathrm{R}}\left(\rho_{n, k}-1\right),
\end{aligned}
$$

$\ddagger$ The kernels in (38), (39) and (40) are not the kernels (8) and (11) used in the recursive definitions of the left and right eigenvectors of $B_{n}(\lambda)$, observe the different types of arguments. 
where $\lambda_{n, k}=-r_{n, k} \omega^{-\rho_{n, k}}, \quad k=1, \ldots, n-1$, are the zeros of the eigenvalue polynomial of $B_{n}(\lambda)$, see (12), and

$$
\begin{gathered}
\Delta_{k}^{+}(\lambda)=\left(\omega^{\rho} / \chi_{k}\right)(\lambda / \omega)^{1-n} \prod_{m=1}^{n-1} F_{m}(\lambda / \omega), \quad \Delta_{k}^{-}(\lambda)=\chi_{k}(\lambda / \omega)^{n-1} F_{n}(\lambda / \omega) \\
\chi_{k}=\frac{r_{n, 0} \tilde{r}_{n-1}}{r_{n} \tilde{r}_{n}}\left(\prod_{\substack{s=1 \\
s \neq k}}^{n-1} y_{n, k}^{n, s} / y_{n, s}^{n, k}\right) \prod_{s=1}^{n-2} y_{n-1, s}^{n, k} .
\end{gathered}
$$

\section{Periodic BBS model for $N=2$}

\subsection{Eigenvalues of transfer matrix for generalized homogeneous Ising model}

In this section we consider in more detail the case of the $N=2$ periodic homogeneous BBS model, where $\omega=-1$. By homogenous we mean that the parameters $a, b, c, d$ and $\varkappa$ each are taken to be independent of the site index. As it was shown in [10] this model is a particular case ("free fermion point") of the generalized Ising model.

In the $N=2$ case, we have $\mathbf{u}_{k}=\sigma_{k}^{z}$ and $\mathbf{v}_{k}=\sigma_{k}^{x}$, where $\sigma_{k}^{z}$ and $\sigma_{k}^{x}$ are Pauli matrices acting on the spin at the $k$-th site. The operator $\mathbf{V}_{n}=\sigma_{1}^{x} \sigma_{2}^{x} \cdots \sigma_{n}^{x}$ is the spin-flip operator. It commutes with the transfer-matrix $\mathbf{t}_{n}(\lambda)$, and we have simply $\mathbf{V}_{n}^{2}=1$. Therefore the eigenvectors of $\mathbf{t}_{n}(\lambda)$ are divided into two sectors according to the eigenvalue $(-1)^{\rho}, \rho \in\{0,1\}$ of $\mathbf{V}_{n}$. If the parameters $a, b, c, d$ and $\varkappa$ are generic, no degeneracies of the eigenvalues occur. The transfer-matrix of the standard Ising model on the finite lattice can be obtained from the $N=2$ BBS integrals of motion $\mathbf{H}_{k}, k=0,1, \ldots, n$, when parameters of the homogeneous $N=2$ BBS model satisfy the relations

$$
a=c, \quad d=b, \quad \varkappa=1 \quad \text { and } \quad \lambda=b / a
$$

(see Section 6.1). In this case degenerations of the spectra occur and in order to distinguish the corresponding eigenvectors one has to use an additional operator which commutes with transfer matrix. In case of the periodic model, such an operator is the operator of translation by one site:

$$
\begin{aligned}
& \mathbf{T}\left|\gamma_{1}\right\rangle_{1} \otimes\left|\gamma_{2}\right\rangle_{2} \otimes \cdots \otimes\left|\gamma_{n}\right\rangle_{n}=\left|\gamma_{n}\right\rangle_{1} \otimes\left|\gamma_{1}\right\rangle_{2} \otimes \cdots \otimes\left|\gamma_{n-1}\right\rangle_{n}, \\
& \mathbf{T} \sigma_{k}^{z, x} \mathbf{T}^{-1}=\sigma_{k+1}^{z, x}, \quad\left[\mathbf{T}, \mathbf{t}_{n}(\lambda)\right]=0, \quad\left[\mathbf{T}, \mathbf{V}_{n}\right]=0 .
\end{aligned}
$$

Our construction of the eigenvectors (38) is obviously non-invariant with respect to translations and we were not able to show that the vectors (38) are also eigenvectors of T. A translational invariant description of the eigenvectors for the generalized Ising model has been given in [18. The description of the spectra of the transfer matrix given in this paper coincides with the description of the eigenvalues obtained in our formalism presented in [1]. This allows to identify the eigenvectors in both descriptions. In particular, the eigenvectors are labelled by quasi-momenta of the excitations $\mathrm{q}= \pm \frac{2 \pi}{n} m$, where $m$ is integer or half-integer depending the eigenvalue $(-1)^{\rho}$ of $\mathbf{V}_{n}$. So the whole space of states decomposes into two sectors according to the value of $\rho$ : 
- NS-sector: $\rho=0$, the eigenstates of $\mathbf{t}_{n}$ have an even number of excitations with quasi-momenta $\mathbf{q} \in \mathrm{NS} \equiv\left\{\frac{2 \pi}{n}\left(\mathbb{Z}_{n}+1 / 2\right)\right\}$. The dimension of this sector is $2^{n-1}$.

- R-sector: $\rho=1$, the eigenstates of $\mathbf{t}_{n}$ have an odd number of excitations with quasi-momenta $\mathrm{q} \in \mathrm{R} \equiv\left\{\frac{2 \pi}{n} \mathbb{Z}_{n}\right\}$. The dimension of this sector is also $2^{n-1}$.

In [1] the eigenvalues $t(\lambda)$ (we suppress the chain-length index $n$ ) were derived from a functional equation which could be written in the form (don't confuse these $A(\mathbf{q})$ etc. with the $A_{n}(\lambda)$ etc. in (2)):

$$
t(\lambda) t(-\lambda)=(-1)^{n} \prod_{\mathbf{q}}\left(A(\mathbf{q}) \lambda^{2}-C(\mathbf{q})+2 i \lambda B(\mathbf{q})\right)
$$

where

$$
\begin{aligned}
& A(\mathbf{q})=a^{2} c^{2}-2 \varkappa a c \cos \mathbf{q}+\varkappa^{2}, \quad B(\mathbf{q})=(a d-b c) \sin \mathbf{q}, \\
& C(\mathbf{q})=1-2(b d / \varkappa) \cos \mathbf{q}+b^{2} d^{2} / \varkappa^{2} .
\end{aligned}
$$

Solving (45), the $2^{n}$ eigenvalues corresponding to the NS- and R-sectors are given by (47) [1, 10, 18] where we have to choose all possible sets of \pm -signs (which we write $(-1)^{\sigma_{\mathrm{q}}}$ with $\left.\sigma_{\mathrm{q}} \in\{0,1\}\right)$ :

$$
\begin{array}{lll}
\mathrm{NS}: & t(\lambda)=\left(a^{n} c^{n}+\varkappa^{n}\right) \prod_{\mathrm{q} \in \mathrm{NS}}\left(\lambda+(-1)^{\sigma_{\mathrm{q}}} s_{\mathrm{q}}\right), & \prod_{\mathrm{q} \in \mathrm{NS}}(-1)^{\sigma_{\mathrm{q}}}=+1, \\
\mathrm{R}: & t(\lambda)=\left(a^{n} c^{n}-\varkappa^{n}\right) \prod_{\mathrm{q} \in \mathrm{R}}\left(\lambda+(-1)^{\sigma_{\mathrm{q}}} s_{\mathrm{q}}\right), & \prod_{\mathrm{q} \in \mathrm{R}}(-1)^{\sigma_{\mathrm{q}}}=-1,
\end{array}
$$

the restriction on the signs follows from (35). We shall also write $\lambda_{\mathbf{q}}=(-1)^{\sigma_{\mathbf{q}}} s_{\mathbf{q}}$, and the amplitudes $s_{\mathrm{q}}$ are given by

$$
s_{\mathbf{q}}=(\sqrt{D(\mathbf{q})}-\mathrm{i} B(\mathbf{q})) / A(\mathbf{q}), \quad D(\mathbf{q})=A(\mathbf{q}) C(\mathbf{q})-B(\mathbf{q})^{2} .
$$

The convention which fixes the sign of $\sqrt{D(\mathrm{q})}$ is given in $(120),(121)$ of [1]. States which have eigenvalues where in (47) there are some minus signs $(-1)^{\sigma_{\mathrm{q}}}=-1$ are said to have excitations with quasi-momenta q. The restriction on the signs means that for the NS (R) sector the number of excitations, i.e. the number of minus signs, must be even (odd).

As an example, the $2^{3}$ eigenstates and eigenvalues for the 3 -site chain $(n=3)$ are:

- NS-sector $(\rho=0)$ :

$$
\begin{array}{ll}
|\rangle_{\mathrm{NS}}(\text { no excitations }) & \rightarrow t(\lambda)=\left(a^{3} c^{3}+\varkappa^{3}\right)\left(\lambda+s_{\pi / 3}\right)\left(\lambda+s_{-\pi / 3}\right)\left(\lambda+s_{\pi}\right), \\
| \pm \pi / 3, \pi\rangle_{\mathrm{NS}}, & \rightarrow t(\lambda)=\left(a^{3} c^{3}+\varkappa^{3}\right)\left(\lambda \mp s_{\pi / 3}\right)\left(\lambda \pm s_{-\pi / 3}\right)\left(\lambda-s_{\pi}\right), \\
|\pi / 3,-\pi / 3\rangle_{\mathrm{NS}}, & \rightarrow t(\lambda)=\left(a^{3} c^{3}+\varkappa^{3}\right)\left(\lambda-s_{\pi / 3}\right)\left(\lambda-s_{-\pi / 3}\right)\left(\lambda+s_{\pi}\right) .
\end{array}
$$

- $\mathrm{R}$-sector $(\rho=1)$ :

$$
\begin{array}{ll}
|0\rangle_{\mathrm{R}} & \rightarrow t(\lambda)=\left(a^{3} c^{3}-\varkappa^{3}\right)\left(\lambda+s_{2 \pi / 3}\right)\left(\lambda+s_{-2 \pi / 3}\right)\left(\lambda-s_{0}\right), \\
| \pm 2 \pi / 3\rangle_{\mathrm{R}} & \rightarrow t(\lambda)=\left(a^{3} c^{3}-\varkappa^{3}\right)\left(\lambda \mp s_{2 \pi / 3}\right)\left(\lambda \pm s_{-2 \pi / 3}\right)\left(\lambda+s_{0}\right), \\
|0,2 \pi / 3,-2 \pi / 3\rangle_{\mathrm{R}} & \rightarrow t(\lambda)=\left(a^{3} c^{3}-\varkappa^{3}\right)\left(\lambda-s_{2 \pi / 3}\right)\left(\lambda-s_{-2 \pi / 3}\right)\left(\lambda-s_{0}\right) .
\end{array}
$$

From [18], it follows that the action of operator of translation $\mathbf{T}$ on the eigenstates gives eigenvalues of the form $e^{\mathrm{i} P}$, where $P$ is the sum of the quasi-momenta of all excitations of the given state. In case of the Ising model when $a d=b c$ and $B(q) \equiv 0$ we have $s_{\mathrm{q}}=s_{-\mathrm{q}}$ : two states which have excitations with quasi-momenta $\mathrm{q}$ and $-\mathrm{q}$ have the same eigenvalues but can be distinguished by the eigenvalue of $\mathbf{T}$. 
The eigenvalues of $\mathbf{t}_{n}(\lambda)$ provide the existence of nontrivial solutions of the systems (41), (42) of homogeneous Baxter equations. These solutions give explicit formulas (38) for the eigenvectors, which form a basis in the space of states of the periodic BBS model for $N=2$.

\subsection{Norms and orthogonality of the eigenvectors of the periodic $N=2 B B S$ model}

Let us fix an eigenvalue polynomial $t(\lambda)$ of $A_{n}(\lambda)+D_{n}(\lambda)$ corresponding to a right eigenvector from the sector $\rho$. In order to find this eigenvector explicitly we have to solve the associated $n-1$ systems $(k=1,2, \ldots, n-1)$ of Baxter equations:

$$
\begin{aligned}
t\left(-r_{n, k}\right) Q_{k}^{\mathrm{R}}(0) & =\left(\Delta_{k}^{+}\left(-r_{n, k}\right)+\Delta_{k}^{-}\left(r_{n, k}\right)\right) Q_{k}^{\mathrm{R}}(1), \\
t\left(r_{n, k}\right) Q_{k}^{\mathrm{R}}(1) & =\left(\Delta_{k}^{+}\left(r_{n, k}\right)+\Delta_{k}^{-}\left(-r_{n, k}\right)\right) Q_{k}^{\mathrm{R}}(0) .
\end{aligned}
$$

Since $t(\lambda)$ is eigenvalue polynomial, it satisfies the functional relation. In [1] it was shown, that the functional relation (45) ensures the existence of non-trivial solutions to (49) with respect to the unknown variables $Q_{k}^{\mathrm{R}}(0)$ and $Q_{k}^{\mathrm{R}}(1)$ for every $k=1,2, \ldots, n-1$. In the $N=2$ case, this means that for every $k$ we have one (in the case of degenerate eigenvalues, possibly zero) independent linear equation. In the case of generic parameters, both hand sides of each equation are non-zero. So we may fix $Q_{k}^{\mathrm{R}}(0)=1 \S$ and obtain two equivalent expressions for $Q_{k}^{\mathrm{R}}(1)$ :

$$
Q_{k}^{\mathrm{R}}(1)=\frac{t\left(-r_{n, k}\right)}{\Delta_{k}^{+}\left(-r_{n, k}\right)+\Delta_{k}^{-}\left(r_{n, k}\right)}=\frac{\Delta_{k}^{+}\left(r_{n, k}\right)+\Delta_{k}^{-}\left(-r_{n, k}\right)}{t\left(r_{n, k}\right)} .
$$

Similarly, for the left eigenvector we have

$$
\begin{aligned}
t\left(-r_{n, k}\right) Q_{k}^{\mathrm{L}}(0) & =(-1)^{n-1}\left(\Delta_{k}^{+}\left(r_{n, k}\right)+\Delta_{k}^{-}\left(-r_{n, k}\right)\right) Q_{k}^{\mathrm{L}}(1), \\
t\left(r_{n, k}\right) Q_{k}^{\mathrm{L}}(1) & =(-1)^{n-1}\left(\Delta_{k}^{+}\left(-r_{n, k}\right)+\Delta_{k}^{-}\left(r_{n, k}\right)\right) Q_{k}^{\mathrm{L}}(0) .
\end{aligned}
$$

Fixing $Q_{k}^{\mathrm{L}}(0)=1$ we obtain

$$
Q_{k}^{\mathrm{L}}(1)=\frac{(-1)^{n-1} t\left(-r_{n, k}\right)}{\Delta_{k}^{+}\left(r_{n, k}\right)+\Delta_{k}^{-}\left(-r_{n, k}\right)}=\frac{\Delta_{k}^{+}\left(-r_{n, k}\right)+\Delta_{k}^{-}\left(r_{n, k}\right)}{(-1)^{n-1} t\left(r_{n, k}\right)} .
$$

Since for generic parameter values we shall have $t\left(r_{n, k}\right) \neq 0$, these explicit formulas give immediately

$$
Q_{k}^{\mathrm{L}}\left(\rho_{n, k}\right) Q_{k}^{\mathrm{R}}\left(\rho_{n, k}\right)=(-1)^{\rho_{n, k}(n-1)} t\left((-1)^{\rho_{n, k}} r_{n, k}\right) / t\left(r_{n, k}\right) .
$$

Eigenvectors in the periodic case are defined by the formula (38). In the case $N=2$ we have a simple inversion relation for the cyclic function $w_{p}(\rho): w_{p}(\rho) w_{p}(\rho-1)=$ $y /(1+x)$. This allows us to use instead of (39) the following kernel

$$
Q^{\mathrm{L}}\left(\boldsymbol{\rho}_{n}^{\prime} \mid \rho, \mathbf{E}\right)=\frac{\prod_{k=1}^{n-1} Q_{k}^{\mathrm{L}}\left(\rho_{n, k}\right)}{\prod_{\substack{s, s^{\prime}=1 \\ s \neq s^{\prime}}}^{n-1} w_{p_{n, s}^{n, s^{\prime}}}\left(\rho_{n, s}-\rho_{n, s^{\prime}}\right)} .
$$

$\S$ When the parameters satisfy the Ising model restrictions, it is not always possible to choose such normalization. 
Form-factors in the BBS model I: Norms and matrix elements

Up to a coefficient, (51) with (38) gives the same eigenvectors of $A_{n}(\lambda)+D_{n}(\lambda)$ as (39). In what follows we shall use only (51). The pairing of $\langle\Phi|$ and $|\Phi\rangle$, which are left and right eigenvectors corresponding to the eigenvalue $t(\lambda)$, gives (we will use the normalization by $\left\langle\tilde{\Psi}_{0, \mathbf{0}} \mid \tilde{\Psi}_{0, \mathbf{0}}\right\rangle$ to get rid of unimportant factors appearing in the formula for the norms)

$\frac{\langle\Phi \mid \Phi\rangle}{\left\langle\tilde{\Psi}_{0, \mathbf{0}} \mid \tilde{\Psi}_{0, \mathbf{0}}\right\rangle}=\sum_{\boldsymbol{\rho}_{n}^{\prime}} \frac{\prod_{k=1}^{n-1} Q_{k}^{\mathrm{L}}\left(\rho_{n, k}\right) Q_{k}^{\mathrm{R}}\left(\rho_{n, k}\right)}{\prod_{l<m}^{n-1}\left(w_{p_{n, l}^{n, m}}\left(\rho_{n, l}-\rho_{n, m}\right) w_{p_{n, m}^{n, l}}\left(\rho_{n, m}-\rho_{n, l}\right)\right)^{2}} \frac{\left\langle\tilde{\Psi}_{\rho, \boldsymbol{\rho}_{n}^{\prime}} \mid \tilde{\Psi}_{\rho, \boldsymbol{\rho}_{n}^{\prime}}\right\rangle}{\left\langle\tilde{\Psi}_{0, \mathbf{0}} \mid \tilde{\Psi}_{0, \mathbf{0}}\right\rangle}$.

where for the norm we have used (20) which leads to

$$
\frac{\left\langle\tilde{\Psi}_{\rho, \boldsymbol{\rho}_{n}^{\prime}} \mid \tilde{\Psi}_{\rho, \boldsymbol{\rho}_{n}^{\prime}}\right\rangle}{\left\langle\tilde{\Psi}_{0, \mathbf{0}} \mid \tilde{\Psi}_{0, \mathbf{0}}\right\rangle}=\frac{\prod_{l<m}^{n-1}\left(r_{n, m}(-1)^{\rho_{n, m}}+r_{n, l}(-1)^{\rho_{n, l}}\right)}{\prod_{l<m}^{n-1}\left(r_{n, m}+r_{n, l}\right)},
$$

where $\mathbf{0}=(0,0, \ldots, 0)$ has $n-1$ components. In the $N=2$ case we have

$$
w_{p}(1)=\frac{y}{1+x}=\frac{1-x}{y}, \quad \frac{1}{\left(w_{p}(1)\right)^{2}}=\frac{1+x}{1-x} .
$$

Therefore for the Fermat point $p_{n, l}^{n, m}=\left(x_{n, l}^{n, m}, y_{n, l}^{n, m}\right)$ with coordinate $x_{n, l}^{n, m}=r_{n, m} / r_{n, l}$ we have

$$
\left(w_{p_{n, l}^{n, m}}\left(\rho_{n, l}-\rho_{n, m}\right)\right)^{-2}=(-1)^{\rho_{n, l}} \frac{\left(r_{n, l}+r_{n, m}\right)}{(-1)^{\rho_{n, l}} r_{n, l}+(-1)^{\rho_{n, m}} r_{n, m}}
$$

and so

$$
\frac{1}{\left(w_{p_{n, l}^{n, m}}\left(\rho_{n, l}-\rho_{n, m}\right) w_{p_{n, m}^{n, l}}\left(\rho_{n, m}-\rho_{n, l}\right)\right)^{2}}=(-1)^{\rho_{n, l}+\rho_{n, m}} \frac{\left(r_{n, l}+r_{n, m}\right)^{2}}{\left((-1)^{\rho_{n, l}} r_{n, l}+(-1)^{\rho_{n, m}} r_{n, m}\right)^{2}} .
$$

Combining all these formulas we get for the left-right overlap of the transfer matrix eigenvectors of the periodic BBS model at $N=2$ :

$$
\frac{\langle\Phi \mid \Phi\rangle}{\left\langle\tilde{\Psi}_{0, \mathbf{0}} \mid \tilde{\Psi}_{0, \mathbf{0}}\right\rangle}=\frac{\prod_{l<m}^{n-1}\left(r_{n, m}+r_{n, l}\right)}{\prod_{l=1}^{n-1} t\left(r_{n, l}\right)} \sum_{\boldsymbol{\rho}_{n}^{\prime}} \frac{\prod_{l=1}^{n-1}(-1)^{\rho_{n, l}} t\left((-1)^{\rho_{n, l}} r_{n, l}\right)}{\prod_{l<m}^{n-1}\left((-1)^{\rho_{n, m}} r_{n, m}+(-1)^{\rho_{n, l}} r_{n, l}\right)} .
$$

Using the same techniques as we used for calculating the norm in the auxiliary problem, we are now able to perform the summations in (53) explicitly: We write the polynomial $t(\lambda)$, equations (36), (47), as

$$
t(\lambda)=\Lambda \prod_{k=1}^{n}\left(\lambda+\mu_{k}\right)
$$

where $\Lambda=a^{n} c^{n}+(-1)^{\rho} \varkappa^{n}$ and $\mu_{k}=\lambda_{\mathbf{q}}$ with $\mathbf{q}=\frac{2 \pi}{n}\left(k+\frac{1-\rho}{2}\right)$. Then the sum in (53) is

$$
\begin{aligned}
& \Lambda^{n-1} \sum_{\boldsymbol{\rho}_{n}^{\prime}} \prod_{l=1}^{n-1}(-)^{\rho_{n, l}} \frac{\prod_{k=1}^{n}\left((-)^{\rho_{n, l}} r_{n, l}+\mu_{k}\right)}{\prod_{j=l+1}^{n-1}\left((-)^{\rho_{n, j}} r_{n, j}+(-)^{\rho_{n, l}} r_{n, l}\right)} \\
& =\frac{\Lambda^{n-1}}{\tilde{r}_{n}^{\prime}} \prod_{l=1}^{n-1} \frac{\prod_{k=1}^{n}\left(r_{n, l}^{2}-\mu_{k}^{2}\right)}{\prod_{m=l+1}^{n-1}\left(r_{n, m}^{2}-r_{n, l}^{2}\right)} \sum_{\rho_{n}^{\prime}} \prod_{l=1}^{n-1}(-)^{\rho_{n, l}} r_{n, l} \frac{\prod_{j=l+1}^{n-1}\left((-)^{\rho_{n, j}} r_{n, j}-(-)^{\rho_{n, l}} r_{n, l}\right)}{\prod_{k=1}^{n}\left(r_{n, l}(-)^{\rho_{n, l}}-\mu_{k}\right)} \\
& =\Lambda^{n-1} \prod_{l=1}^{n-1} \frac{\prod_{k=1}^{n}\left(r_{n, l}^{2}-\mu_{k}^{2}\right)}{\prod_{m=l+1}^{n-1}\left(r_{n, m}^{2}-r_{n, l}^{2}\right)} 2^{n-1} \tilde{r}_{n}^{\prime} \prod_{k<k^{\prime}} \frac{\mu_{k}^{2}-\mu_{k^{\prime}}^{2}}{\mu_{k}-\mu_{k^{\prime}}} \frac{\prod_{l<l^{\prime}}^{n-1}\left(r_{n, l}^{2}-r_{n, l^{\prime}}^{2}\right)}{\prod_{l=1}^{n} \prod_{l^{\prime}=1}^{n-1}\left(\mu_{l}^{2}-r_{n, l^{\prime}}^{2}\right)} \\
& =\Lambda^{n-1} 2^{n-1} \tilde{r}_{n}^{\prime} \prod_{k<k^{\prime}}\left(\mu_{k}+\mu_{k^{\prime}}\right), \quad \tilde{r}_{n}^{\prime}=r_{n, 1} r_{n, 2} \ldots r_{n, n-1} .
\end{aligned}
$$


In the second line the sum takes just the form (79) which we had for $S_{n+1}$ with $N=2$ and $\mathbf{a}^{(n+1)}=\left(r_{n, 1}(-)^{\rho_{n, 1}}, \ldots, r_{n, n-1}(-)^{\rho_{n, n-1}}\right)$ and $\mathbf{b}^{(n+1)}=\left(\mu_{1}, \ldots, \mu_{n}\right)$. In the last two lines we have used the result (80) for $S_{n+1}$ derived in the Appendix. So we obtain the norm of the general periodic state vector

$$
\frac{\langle\Phi \mid \Phi\rangle}{\left\langle\tilde{\Psi}_{0, \mathbf{0}} \mid \tilde{\Psi}_{0, \mathbf{0}}\right\rangle}=2^{n-1} \tilde{r}_{n}^{\prime} \frac{\prod_{l<m}^{n-1}\left(r_{n, m}+r_{n, l}\right)}{\prod_{k=1}^{n} \prod_{l=1}^{n-1}\left(r_{n, l}+\mu_{k}\right)} \prod_{i<j}^{n}\left(\mu_{i}+\mu_{j}\right) .
$$

If the spectrum of the model is not degenerate, the overlap of the eigenvectors corresponding to different eigenvalues is zero. In case of degeneration, as it happens in the case of the parameterization $a=c, b=d$ and $\varkappa=1$, corresponding to the Ising model, one should be careful using (56). In Section 6 this case will be discussed in detail.

\subsection{Matrix elements between eigenvectors of the periodic $N=2 B B S$ model}

In Theorem 2 we found the action of $\mathbf{u}_{n}$ on an eigenvector $\left|\Psi_{\boldsymbol{\rho}_{n}}\right\rangle$ of $B_{n}(\lambda)$ : the result is a linear combination of the original vector plus a sum of vectors which each have one component of $\boldsymbol{\rho}_{n}$ raised. In order to get the matrix elements of $\mathbf{u}_{n}$ in the periodic model, we first obtain the matrix elements between Fourier transformed eigenvectors of $B_{n}(\lambda)$ defined in (35)

$$
\left\langle\tilde{\Psi}_{\rho, \boldsymbol{\rho}_{n}^{\prime}}\left|=\left\langle\Psi_{0, \boldsymbol{\rho}_{n}^{\prime}}\left|+(-)^{\rho}\left\langle\Psi_{1, \boldsymbol{\rho}_{n}^{\prime}}|, \quad| \tilde{\Psi}_{\rho, \boldsymbol{\rho}_{n}^{\prime}}\right\rangle=\right| \Psi_{0, \boldsymbol{\rho}_{n}^{\prime}}\right\rangle+(-)^{\rho}\right| \Psi_{1, \boldsymbol{\rho}_{n}^{\prime}}\right\rangle .
$$

Using (30), the following $n$-site matrix elements of $\mathbf{u}_{n}$ are non-zero

$$
\begin{aligned}
& \frac{\left\langle\tilde{\Psi}_{1, \boldsymbol{\rho}_{n}^{\prime}}\left|\mathbf{u}_{n}\right| \tilde{\Psi}_{0, \boldsymbol{\rho}_{n}^{\prime}}\right\rangle}{\left\langle\tilde{\Psi}_{0, \boldsymbol{\rho}_{n}^{\prime}} \mid \tilde{\Psi}_{0, \boldsymbol{\rho}_{n}^{\prime}}\right\rangle}=\frac{\tilde{x}_{n}}{x_{n}}(-1)^{\tilde{\rho}_{n}^{\prime}}+\frac{y_{n, 0} y_{n}}{x_{n}}=\frac{a_{n}}{\tilde{r}_{n}}(-1)^{\tilde{\rho}_{n}^{\prime}}+\frac{\varkappa_{1} \varkappa_{2} \cdots \varkappa_{n-1} b_{n}}{r_{n, 0}}, \\
& \frac{\left\langle\tilde{\Psi}_{0, \boldsymbol{\rho}_{n}^{\prime}}\left|\mathbf{u}_{n}\right| \tilde{\Psi}_{1, \boldsymbol{\rho}_{n}^{\prime}}\right\rangle}{\left\langle\tilde{\Psi}_{0, \boldsymbol{\rho}_{n}^{\prime}} \mid \tilde{\Psi}_{0, \boldsymbol{\rho}_{n}^{\prime}}\right\rangle}=\frac{\tilde{x}_{n}}{x_{n}}(-1)^{\tilde{\rho}_{n}^{\prime}}-\frac{y_{n, 0} y_{n}}{x_{n}}=\frac{a_{n}}{\tilde{r}_{n}}(-1)^{\tilde{\rho}_{n}^{\prime}}-\frac{\varkappa_{1} \varkappa_{2} \cdots \varkappa_{n-1} b_{n}}{r_{n, 0}}, \\
& \frac{\left\langle\tilde{\Psi}_{1, \boldsymbol{\rho}_{n}^{+k}}\left|\mathbf{u}_{n}\right| \tilde{\Psi}_{0, \boldsymbol{\rho}_{n}^{\prime}}\right\rangle}{\left\langle\tilde{\Psi}_{0, \boldsymbol{\rho}_{n}^{\prime}} \mid \tilde{\Psi}_{0, \boldsymbol{\rho}_{n}^{\prime}}\right\rangle}=\frac{\left\langle\tilde{\Psi}_{0, \boldsymbol{\rho}_{n}^{\prime+k}}\left|\mathbf{u}_{n}\right| \tilde{\Psi}_{1, \boldsymbol{\rho}_{n}^{\prime}}\right\rangle}{\left\langle\tilde{\Psi}_{0, \boldsymbol{\rho}_{n}^{\prime}} \mid \tilde{\Psi}_{0, \boldsymbol{\rho}_{n}^{\prime}}\right\rangle}= \\
& =(-1)^{\tilde{\rho}_{n}^{\prime}} \frac{\tilde{r}_{n-1} a_{n} b_{n} c_{n}}{r_{n} r_{n, 0}}\left(1+\frac{(-1)^{\rho_{n, k}} d_{n}}{\varkappa_{n} c_{n} r_{n, k}}\right) \frac{\prod_{l=1}^{n-2} y_{n-1, l}^{n, k}}{\prod_{s \neq k}\left(r_{n, k}(-1)^{\rho_{n, k}}+r_{n, s}(-1)^{\left.\rho_{n, s}\right)}\right.},
\end{aligned}
$$

where $\tilde{\rho}_{n}^{\prime}=\rho_{n, 1}+\rho_{n, 2}+\cdots+\rho_{n, n-1}$. Note, since $\mathbf{u}_{n}$ anti-commutes with $\mathbf{V}_{n}$, all matrix elements of $\mathbf{u}_{n}$ between the vectors from the same sector $\rho$ are zero.

Then by (38) we pass to periodic eigenstates transforming by the solutions $Q$ of the Baxter equations. Let $\left\langle\Phi_{0}\right|$ be a left eigenvector of the transfer-matrix $\mathbf{t}_{n}(\lambda)$ with $\rho=0$ and $\left|\Phi_{1}\right\rangle$ be a right eigenvector with $\rho=1$. Let $Q_{k}^{L(0)}\left(\rho_{n, k}\right)$ and $Q_{k}^{R(1)}\left(\rho_{n, k}\right)$ be the solutions of Baxter equation corresponding to these two eigenvectors. Then for the matrix element after some simplification we have

$$
\frac{\left\langle\Phi_{0}\left|\mathbf{u}_{n}\right| \Phi_{1}\right\rangle}{\left\langle\tilde{\Psi}_{0, \mathbf{0}} \mid \tilde{\Psi}_{0, \mathbf{0}}\right\rangle}=\sum_{\boldsymbol{\rho}_{n}^{\prime}}(-1)^{n \tilde{\rho}_{n}^{\prime}} \prod_{l<m}^{n-1} \frac{r_{n, l}+r_{n, m}}{(-1)^{\rho_{n, l}} r_{n, l}+(-1)^{\rho_{n, m}} r_{n, m}} \times
$$


Form-factors in the BBS model I: Norms and matrix elements

$$
\begin{aligned}
& \times\left(\prod_{l=1}^{n-1} Q_{l}^{L(0)}\left(\rho_{n, l}\right) Q_{l}^{R(1)}\left(\rho_{n, l}\right)\left(\frac{a_{n}}{\tilde{r}_{n}}(-1)^{\tilde{\rho}_{n}^{\prime}}-\frac{\varkappa_{1} \varkappa_{2} \cdots \varkappa_{n-1} b_{n}}{r_{n, 0}}\right)+\right. \\
& +\sum_{k=1}^{n-1} Q_{k}^{L(0)}\left(\rho_{n, k}+1\right) Q_{k}^{R(1)}\left(\rho_{n, k}\right) \prod_{l \neq k}^{n-1} Q_{l}^{L(0)}\left(\rho_{n, l}\right) Q_{l}^{R(1)}\left(\rho_{n, l}\right) \cdot r_{n, k}^{n-1} \chi_{k}(-1)^{(n-1) \rho_{n, k}} \times \\
& \left.\quad \times \frac{a_{n} b_{n} c_{n}}{r_{n, 0}}\left(1+\frac{(-1)^{\rho_{n, k}} d_{n}}{\varkappa_{n} c_{n} r_{n, k}}\right) \frac{1}{\prod_{s \neq k}\left(r_{n, k}(-1)^{\rho_{n, k}}-r_{n, s}(-1)^{\left.\rho_{n, s}\right)}\right.}\right)
\end{aligned}
$$

The product on the right hand side of the first line comes from the change of normalization from (57) to (158) by the factor $\left\langle\tilde{\Psi}_{0, \boldsymbol{\rho}^{\prime}} \mid \tilde{\Psi}_{0, \boldsymbol{\rho}^{\prime}}\right\rangle /\left\langle\tilde{\Psi}_{0, \mathbf{0}} \mid \tilde{\Psi}_{0, \mathbf{0}}\right\rangle$, which can simply be read off from (20) with $C_{n}$ cancelling.

We have not yet been able to perform the summation over $\boldsymbol{\rho}_{n}^{\prime}$ in (58) for general parameters of the BBS-model. However, for the homogenous Ising case (44), we can show that (58) can be put into a fully factorized form, although in this case special complications appear from the coincidence of the zeros of the transfer matrix with the polynomial zeros of $B_{n}(\lambda)$. In the next section we give the result of this summation, which proves a formula for the matrix elements of the spin operator (form-factors) on a finite lattice conjectured [14, 15] by A. Bugrij and O. Lisovyy. Details of the proof which is a new result for the Ising model, and the comparison with the notation of [14, 15] are relegated to the sequel article [16].

\section{The homogeneous Ising model}

\subsection{Relation to the standard Ising model}

In this section we restrict the parameters of the homogeneous $N=2 \mathrm{BSS}$ model to be $a=c, b=d$ and $\varkappa=1$. So the cyclic $L$-operator (11) reduces to

$$
L_{k}(\lambda)=\left(\begin{array}{cc}
1+\lambda \mathbf{v}_{k} & \lambda \mathbf{u}_{k}\left(a-b \mathbf{v}_{k}\right) \\
\mathbf{u}_{k}\left(a-b \mathbf{v}_{k}\right) & \lambda a^{2}+\mathbf{v}_{k} b^{2}
\end{array}\right) .
$$

Let us make the special choice of the spectral parameter $\lambda=b / a$ as in (44). Then the $L$-operator degenerates and we get

$$
L_{k}(b / a)=\left(1+\mathbf{v}_{k} b / a\right)\left(\begin{array}{cc}
1 & b \mathbf{u}_{k} \\
a \mathbf{u}_{k} & a b
\end{array}\right)=\left(1+\mathbf{v}_{k} b / a\right)\left(\begin{array}{c}
1 \\
a \mathbf{u}_{k}
\end{array}\right)\left(\begin{array}{cc}
1 & b \mathbf{u}_{k}
\end{array}\right) .
$$

At this point the transfer matrix is

$\mathbf{t}_{n}(b / a)=\operatorname{tr} L_{1}(b / a) L_{2}(b / a) \cdots L_{n}(b / a)=\prod_{k=1}^{n}\left(1+\mathbf{v}_{k} \cdot b / a\right) \cdot \prod_{k=1}^{n}\left(1+\mathbf{u}_{k} \mathbf{u}_{k+1} \cdot a b\right)$.

Recall that due to the periodic boundary conditions $\mathbf{u}_{n+k} \equiv \mathbf{u}_{k}$. Using

$\exp \left(K_{x} \mathbf{u}_{k} \mathbf{u}_{k+1}\right)=\cosh K_{x}\left(1+\mathbf{u}_{k} \mathbf{u}_{k+1} \tanh K_{x}\right), \exp \left(K_{x}^{*} \mathbf{v}_{k}\right)=\cosh K_{x}^{*}\left(1+\mathbf{v}_{k} \tanh K_{x}^{*}\right)$ 
and writing $\mathbf{u}_{k}=\sigma_{k}^{z}$ and $\mathbf{v}_{k}=\sigma_{k}^{x}$, it is easy to identify $\mathbf{t}_{n}(b / a)$ with the standard Ising transfer-matrix:

$$
\begin{aligned}
& \mathbf{t}_{\text {Ising }}=\exp \left(\sum_{k=1}^{n} K_{x}^{*} \sigma_{k}^{x}\right) \exp \left(\sum_{k=1}^{n} K_{x} \sigma_{k}^{z} \sigma_{k+1}^{z}\right), \\
& e^{-2 K_{y}}=\tanh K_{x}^{*}=\frac{b}{a}, \quad \tanh K_{x}=a b,
\end{aligned}
$$

where $K_{x, y}$ and $K_{x, y}^{*}$ are coupling constants of Ising and dual Ising models on the square lattice along the $X$ and $Y$ axes, respectively.

The eigenvectors are obtained by the method of separation of variables, they do not depend on $\lambda$. In what follows we shall not fix $\lambda=b / a$ and so we consider a family of models depending on $\lambda, a$ and $b$. This family includes the Ising model at $\lambda=b / a$ and the eigenvectors obtained by SoV are eigenvectors of the Ising transfer matrix too. Let us note that this $L$-operator formulation can be extended [10] to a larger family of Ising-like models, giving a possibility to use SoV for finding explicit formulas for the eigenvectors of corresponding transfer-matrices.

In (45) and (47) from [1] we already quoted the functional equation and the eigenvalues of the general homogeneous $N=2 \mathrm{BBS}$ model. Introducing

$\mathcal{T}\left(\lambda^{2}\right)=1+b^{2} d^{2} / \varkappa^{2}-\lambda^{2}\left(\varkappa^{2}+a^{2} c^{2}\right), \quad F(\lambda)=(b-a \varkappa \lambda)(\lambda c+d / \varkappa)$,

the equation $A(\mathbf{q}) \lambda_{\mathbf{q}}^{2}-C(\mathbf{q})+2 i \lambda_{\mathbf{q}} B(\mathbf{q})=0$ can be written as

$$
\mathcal{T}\left(s_{\mathbf{q}}^{2}\right)=e^{\mathrm{iq}} F\left(s_{\mathbf{q}}\right)+e^{-\mathrm{iq}} F\left(-s_{\mathbf{q}}\right) .
$$

The solution of (63) is given by (48) where $\mathrm{q}= \pm \frac{\pi}{n} m$, with $m$ even for the R-sector $(\rho=1)$ and $m$ odd for the NS-sector $(\rho=0)$.

The equations for the amplitudes $r_{n, k}$ of the roots of the eigenvalue polynomial of the operator $B_{n}(\lambda)$ of equation (9) were derived in equation (A7) of [1]

$\mathcal{T}\left(r_{n, k}^{2}\right)^{2}=4 \cos ^{2}\left(q_{n, k}\right) F\left(r_{n, k}\right) F\left(-r_{n, k}\right), \quad q_{n, k}=\pi k / n, \quad k=1,2, \ldots, n-1$.

In the case of the Ising model parametrization (44) we have $F(\lambda)=b^{2}-a^{2} \lambda^{2}$, and (63) and (64) reduce to $\mathcal{T}\left(s_{\mathbf{q}}^{2}\right)=2 \cos (\mathbf{q}) F\left(s_{\mathbf{q}}\right)$ and $\mathcal{T}\left(r_{n, k}^{2}\right)^{2}=4 \cos ^{2}\left(q_{n, k}\right) F^{2}\left(r_{n, k}\right)$, respectively. Then the solutions of these equations are:

$$
s_{\mathrm{q}}=\sqrt{\frac{b^{4}-2 b^{2} \cos \mathrm{q}+1}{a^{4}-2 a^{2} \cos \mathrm{q}+1}}, \quad r_{n, k}=\sqrt{\frac{b^{4}-2 b^{2} \cos q_{n, k}+1}{a^{4}-2 a^{2} \cos q_{n, k}+1}},
$$

where the momentum $\mathrm{q}$ in both NS- and R-sectors takes the values $\left\{0, \pi, \pm q_{n, k}\right\}$ with $0<q_{n, k}<\pi$, in particular $q_{n, k} \neq 0, \pi$. So we have

$$
r_{n, k}=s_{q_{n, k}}=s_{-q_{n, k}}, \quad s_{0}=\frac{b^{2}-1}{a^{2}-1}, \quad s_{\pi}=\frac{b^{2}+1}{a^{2}+1} .
$$

The possible coincidence in the Ising case of the two quite different parameters: the zeros $r_{n, k}$ of the polynomial $B_{n}(\lambda)$ and the zeros $s_{\mathrm{q}}$ of transfer matrix will create some peculiarities when in the following we set out to solve the Baxter equations.

\|In [1] we used $\phi_{n, k} / 2$ instead of $q_{n, k}$. 


\subsection{Solution of the Baxter equations}

We have seen that in the Ising case (44) the eigenvectors and eigenvalues of $\mathbf{t}_{n}(\lambda)$ decompose into two sectors $\rho=0,1$. With (44) in addition we have also $F(\lambda)=F(-\lambda)$ and the Baxter equations (41), (42) for left and right kernels become identical. Omitting the superscripts $L$ and $R$ on $Q_{k}$ and writing $\lambda_{n, k}=-(-1)^{\rho_{n, k}} r_{n, k}, \quad \rho_{n, k}=0,1$ we obtain:

$t\left(\lambda_{n, k}\right) Q_{k}\left(\rho_{n, k}\right)=\left(\frac{(-1)^{\rho} F^{n-1}\left(\lambda_{n, k}\right)}{\left(\lambda_{n, k}\right)^{n-1} \chi_{k}}+\left(-\lambda_{n, k}\right)^{n-1} \chi_{k} F\left(\lambda_{n, k}\right)\right) Q_{k}\left(\rho_{n, k}+1\right)$.

In order to solve these Baxter equations for a given sector $\rho$, we need $t\left( \pm r_{n, k}\right)$. Now from (47) we have

$$
t(\lambda)=\left(a^{2 n}+(-1)^{\rho}\right) \prod_{\mathbf{q}}\left(\lambda+\lambda_{\mathbf{q}}\right), \quad \lambda_{\mathbf{q}}=(-1)^{\sigma_{\mathrm{q}}} s_{\mathbf{q}},
$$

and we see that $t_{n}\left( \pm r_{n, k}\right)$ vanishes if $\lambda_{\mathrm{q}}=\mp r_{n, k}$. When $\rho$ and $k$ have the same parity $(-)^{\rho}=(-)^{k}$ the quasi-momenta $\mathbf{q}$ which describe the spectrum $\lambda_{\mathbf{q}}$ of the transfer matrix do not coincide with any $q_{n, k}$ and the value of the transfer matrix $t\left( \pm r_{n, k}\right)$ does not vanish. If $\rho$ and $k$ have different parities: $(-)^{\rho}=(-)^{k+1}$ this could happen. Below, when we solve the Baxter equations we shall treat these cases separately.

Moreover, as we can see from (66) , for the Ising parameterization the amplitudes $s_{q_{n, k}}=s_{-q_{n, k}}$ coincide. This leads to a degeneracy of eigenvalues of $\mathbf{t}_{n}(\lambda)$ : an eigenstate with $\mathbf{q}=q_{n, k}$ excited and $\mathbf{q}=-q_{n, k}$ not excited has the same eigenvalues as the eigenstate with $-q_{n, k}$ excited and $q_{n, k}$ not excited: both have the same factor $\left(\lambda^{2}-s_{q_{n, k}}^{2}\right)$ in the eigenvalue polynomial. The degeneracy of eigenvalues of the transfer-matrix can lead potentially to the following problem: the functional relation guarantees, of course, the existence of a non-trivial solution of the Baxter equations for any particular eigenvalue-polynomial, but we need in our case two independent solutions. Fortunately, due to the coincidence $s_{\mathrm{q}= \pm q_{n, k}}=r_{n, k}$ both sides of the Baxter equations for the unknowns $Q_{k}(0)$ and $Q_{k}(1)$ become zero and we can build a two-dimensional solutions space.

The compatibility condition following from (67) is

$t\left(-r_{n, k}\right) t\left(r_{n, k}\right)=(-1)^{n-1}\left(\frac{(-1)^{\rho} F^{n-1}\left(r_{n, k}\right)}{\left(r_{n, k}\right)^{n-1} \chi_{k}}+\left(-r_{n, k}\right)^{n-1} \chi_{k} F\left(r_{n, k}\right)\right)^{2}$,

where $t(\lambda)$ is an eigenvalue from the sector $\rho$. If $k$ is such that $(-1)^{k}=(-1)^{\rho+1}$, then the quasi-momentum $\mathrm{q}=q_{n, k}$ belongs to the sector $\rho$ and for $r_{n, k}=s_{q_{n, k}}$ we have $t\left(-r_{n, k}\right) t\left(r_{n, k}\right)=0$. This implies a relation not depending on a particular $t(\lambda)$ and its $\rho$ :

$$
\chi_{k}^{2} r_{n, k}^{2(n-1)}=(-1)^{n+k+1} F^{n-2}\left(r_{n, k}\right) .
$$

In order to find the eigenvector corresponding to $t(\lambda)$ from the sector $\rho$ we have to find all the $Q_{k}\left(\rho_{n, k}\right)$ for all $k$ solving the Baxter equations. We need to distinguish the following four cases with respect to the value $k$ :

(i) $(-1)^{\rho}=(-1)^{k}$. In this case both $t\left(r_{n, k}\right) \neq 0$ and $t\left(-r_{n, k}\right) \neq 0$. So we may fix $Q_{k}(0)=1$ and, using (69) we obtain

$$
Q_{k}(1)=\frac{(-1)^{n-1} t\left(-r_{n, k}\right)}{2 \chi_{k} r_{n, k}^{n-1} F\left(r_{n, k}\right)} \text {. }
$$


The other three cases correspond to $(-1)^{\rho}=(-1)^{k-1}$ so that the big brackets on the right-hand sides of the Baxter equations are zero due to (69).

(ii) $t\left(r_{n, k}\right) \neq 0, t\left(-r_{n, k}\right)=0$ : The eigenvalue polynomial $t(\lambda)$ contains the factor $\left(\lambda+r_{n, k}\right)^{2}$ (both momenta $\mathbf{q}= \pm q_{n, k}$ are not excited, i.e. both not in the spectrum) and we have

$$
Q_{k}(0)=1, \quad Q_{k}(1)=0
$$

(iii) $t\left(r_{n, k}\right)=0, t\left(-r_{n, k}\right) \neq 0: t(\lambda)$ contains the factor $\left(\lambda-r_{n, k}\right)^{2}$ (both momenta $\mathbf{q}= \pm q_{n, k}$ are excited) and we have

$$
Q_{k}(0)=0, \quad Q_{k}(1)=1 .
$$

(iv) both $t\left(r_{n, k}\right)=0$ and $t\left(-r_{n, k}\right)=0$. This case happens when only one of the two quasi-momenta $\mathbf{q}= \pm q_{n, k}$ is excited, so that $t(\lambda)$ contains a factor $\left(\lambda^{2}-r_{n, k}^{2}\right)$. As we explained above, this eigenvalue is degenerate. In this case both hand sides of both Baxter equations are zero. In principle, we can choose any two independent solutions for Baxter equations, but, in general, they will not give us eigenvectors of operator of translation T. Since our construction of the eigenvectors (38) is obviously non-invariant with respect to translations it is unclear how using the direct action of $\mathbf{T}$ one can choose solutions of the Baxter equations which give such eigenvectors. We take another way: In order to obtain eigenvectors of transfer matrix which are eigenvectors also of the translation operator, we shall first lift the degeneracy by starting with parameters such that $a d-b c=\eta$ (keeping $\varkappa=1$ ) with $\eta$ small but finite. At the end we shall take the limit $\eta \rightarrow 0$. Observe that for $\eta \neq 0$ (49) and (50) are different. From (49) we get

$$
Q_{k}^{\mathrm{R}}(0)=1, \quad Q_{k}^{\mathrm{R}}(1)=\frac{t\left(-r_{n, k}\right)}{(-1)^{\rho} /\left(\chi_{k}\left(r_{n, k}\right)^{n-1}\right) F^{n-1}\left(r_{n, k}\right)+(-1)^{n-1} \chi_{k}\left(r_{n, k}\right)^{n-1} F\left(-r_{n, k}\right)} .
$$

When $\eta \rightarrow 0$, both numerator and denominator of $Q_{k}^{\mathrm{R}}(1)$ tend to 0 . Let us find their leading (in fact, linear) terms in $\eta \rightarrow 0$. For the denominator we have for $\eta \rightarrow 0$

$$
\frac{(-1)^{\rho} F^{n-1}\left(r_{n, k}\right)}{\chi_{k}\left(r_{n, k}\right)^{n-1}}+(-1)^{n-1} \chi_{k}\left(r_{n, k}\right)^{n-1} F\left(-r_{n, k}\right) \sim \eta(-1)^{n+1} n r_{n, k} \chi_{k}\left(r_{n, k}\right)^{n-1},
$$

where we used the relation (valid for arbitrary $\eta$ )

$$
F^{n-2}\left(r_{n, k}\right) F^{n-2}\left(-r_{n, k}\right)=\chi_{k}^{4} r_{n, k}^{4(n-1)} .
$$

Let us write the transfer-matrix eigenvalue polynomial (68) as

$$
t(\lambda)=t_{\check{\mathbf{q}}_{k}}(\lambda)\left(\lambda-s_{\check{\mathrm{q}}_{k}}\right)\left(\lambda+s_{-\check{\mathrm{q}}_{k}}\right),
$$

where $\check{\mathrm{q}}_{k}=(-1)^{\sigma_{q_{n, k}+1}} q_{n, k}$ is the value of the excited quasi-momentum, so that

$$
t\left(-r_{n, k}\right)=t_{\check{\mathbf{q}}_{k}}\left(-r_{n, k}\right)\left(r_{n, k}-s_{-\breve{\mathrm{q}}_{k}}\right)\left(r_{n, k}+s_{\check{\mathrm{q}}_{k}}\right) .
$$

Using $A(\mathbf{q})\left(\lambda-s_{-\mathbf{q}}\right)\left(\lambda+s_{\mathbf{q}}\right)=A(\mathbf{q}) \lambda^{2}-C(\mathbf{q})-2$ i $B(\mathbf{q}) \lambda, \quad B(\mathbf{q})=\eta \sin \mathbf{q}$ and $A\left(\check{\mathrm{q}}_{k}\right) r_{n, k}^{2}-C\left(\check{\mathrm{q}}_{k}\right) \sim o(\eta)$ (due to (46) and (48) $)$ at $\eta \rightarrow 0$ ) we get

$t\left(-r_{n, k}\right) \sim-t_{\check{\mathbf{q}}_{k}}\left(-r_{n, k}\right) 2 \mathrm{i} B\left(\check{\mathbf{q}}_{k}\right) r_{n, k} / A\left(\check{\mathbf{q}}_{k}\right)=-t_{\check{\mathbf{q}}_{k}}\left(-r_{n, k}\right) 2 \mathrm{i} \sin \left(\check{\mathbf{q}}_{k}\right) r_{n, k} \eta / A\left(\check{\mathbf{q}}_{k}\right)$

I In this case we can't normalize $Q_{k}(0)=1$. 
at $\eta \rightarrow 0$, where $A(\mathbf{q})=a^{4}-2 a^{2} \cos \mathbf{q}+1$. Finally,

$$
Q_{k}^{\mathrm{R}}(0)=1, \quad Q_{k}^{\mathrm{R}}(1)=\frac{(-1)^{n+\sigma_{q_{n, k}}+1} 2 \mathrm{i} \sin \left(q_{n, k}\right) t_{\check{\mathrm{q}}_{k}}\left(-r_{n, k}\right)}{n \chi_{k} r_{n, k}^{n-1} A\left(q_{n, k}\right)} .
$$

Using a similar limiting procedure for the Baxter equation (50) we get

$$
Q_{k}^{\mathrm{L}}(0)=1, \quad Q_{k}^{\mathrm{L}}(1)=-Q_{k}^{\mathrm{R}}(1) .
$$

Although the Ising Baxter equations were the same for $Q^{L}$ and $Q^{R}$, in this case, since we take the limit of the general non-hermitian BBS case, $Q_{k}^{L}(1)$ and $Q_{k}^{R}(1)$ come out different.

\subsection{Norms and factorized matrix elements for the homogeneous Ising model}

In this Subsection we state a new factorized formula for the matrix element of the spin operator in the Ising model on a finite lattice. Let $\left|\Phi_{0}\right\rangle$ and $\left|\Phi_{1}\right\rangle$ be two eigenvectors of the periodic Ising model from the sectors $\rho=0$ and $\rho=1$, respectively. First let us consider the case when these states do not contain fermionic excitations with momenta $\mathbf{q}=q_{n, k}$ and $\mathbf{q}=-q_{n, k}$ simultaneously, so that the eigenvalue polynomials do not contain a factor $\left(\lambda-r_{n, k}\right)^{2}$. We introduce the subset $\mathcal{D} \subset\{1,2, \ldots, n-1\}$ of indices $k$ for which the eigenvalue polynomials of $\left|\Phi_{0}\right\rangle$ and $\left|\Phi_{1}\right\rangle$ contain the factor $\left(\lambda^{2}-r_{n, k}^{2}\right)$, i.e. for which we have the case (iv) of Subsection 6.2, Denote by $|\mathcal{D}|$ the size of the set $\mathcal{D}$.

Let $\delta=1$ if $\sigma_{0}=\sigma_{\pi}$ and $\delta=0$ otherwise. We also use the short notations: $\lambda_{0}=(-1)^{\sigma_{0}} s_{0}, \lambda_{\pi}=(-1)^{\sigma_{\pi}} s_{\pi}$ and $\hat{q}_{k}=(-1)^{\sigma_{q_{n, k}}+k} q_{n, k}$. Now the matrix element is given by the factorized formula:

$$
\begin{aligned}
& \frac{\left\langle\Phi_{0}\left|\mathbf{u}_{n}\right| \Phi_{1}\right\rangle\left\langle\Phi_{1}\left|\mathbf{u}_{n}\right| \Phi_{0}\right\rangle}{\left\langle\tilde{\Psi}_{0, \mathbf{0}} \mid \tilde{\Psi}_{0, \mathbf{0}}\right\rangle^{2}}=\left(\lambda_{\pi}^{2}-\lambda_{0}^{2}\right)^{(|\mathcal{D}|-\delta) / 2}\left(\lambda_{0}+\lambda_{\pi}\right)^{\delta} \times \\
& \times \prod_{k \in \mathcal{D}} \frac{2 r_{n, k}}{\left(r_{n, k}+\lambda_{0}\right)\left(r_{n, k}+\lambda_{\pi}\right)} \prod_{\substack{k<m \\
k, m \in \mathcal{D}}} \frac{r_{n, k}+r_{n, m}}{r_{n, k}-r_{n, m}} \prod_{\substack{k<m \\
k, m \in \mathcal{D}}} \frac{\sin \frac{1}{2}\left(\hat{q}_{k}-\hat{q}_{m}\right)}{\sin \frac{1}{2}\left(\hat{q}_{k}+\hat{q}_{m}\right)} .
\end{aligned}
$$

The proof of (70) is given in our sequel article [16].

In order to compare (70) to the results obtained by A. Bugrij and O. Lisovyy [14, 15] we have to calculate instead of (70) the ratio

$$
\frac{\left\langle\Phi_{0}\left|\mathbf{u}_{n}\right| \Phi_{1}\right\rangle\left\langle\Phi_{1}\left|\mathbf{u}_{n}\right| \Phi_{0}\right\rangle}{\left\langle\Phi_{0} \mid \Phi_{0}\right\rangle\left\langle\Phi_{1} \mid \Phi_{1}\right\rangle}
$$

which has the advantage to be independent of the particular normalization of $\left|\Phi_{0}\right\rangle$ and $\left|\Phi_{1}\right\rangle$. To do this we have to divide (170) by

$$
\left\langle\Phi_{0} \mid \Phi_{0}\right\rangle\left\langle\Phi_{1} \mid \Phi_{1}\right\rangle /\left\langle\tilde{\Psi}_{0, \mathbf{0}} \mid \tilde{\Psi}_{0, \mathbf{0}}\right\rangle^{2} .
$$

The expression for (72) was obtained in (56) for generic parameters. In the case of the Ising model the norms of the degenerated states cannot be directly obtained from (56)), because the numerator and denominator contain zeros. When degenerated states appear, that is when $\mathcal{D}$ is not empty, we have to be careful in using the formula (56) for the norms and use l'Hôpital's rule. In order to implement l'Hôpital's rule we proceed 
similarly to what we did in case (iv) of Section 6.2 for finding the solutions of Baxter equations: we go off the Ising point taking $a d-b c=\eta$ to be small finite and keep terms linear in $\eta$. Let $\check{\mathrm{q}}_{l}=(-1)^{\sigma_{q_{n, l}+1}} q_{n, l}$ for $l \in \mathcal{D}$. Then the coefficient of $\lambda$ in

$$
A\left(\check{\mathrm{q}}_{l}\right)\left(\lambda-s_{\check{\mathrm{q}}_{l}}\right)\left(\lambda+s_{-\check{\mathrm{q}}_{l}}\right)=A\left(\check{\mathrm{q}}_{l}\right) \lambda^{2}-C\left(\check{\mathrm{q}}_{l}\right)+2 \mathrm{i} B\left(\check{\mathrm{q}}_{l}\right) \lambda
$$

gives $-s_{\check{\mathbf{q}}_{l}}+s_{-\check{\mathrm{q}}_{l}}=2 \mathrm{i} B\left(\check{\mathrm{q}}_{l}\right) / A\left(\check{\mathrm{q}}_{l}\right)$. Due to $A\left(\check{\mathrm{q}}_{l}\right) r_{n, l}^{2}-C\left(\check{\mathrm{q}}_{l}\right) \sim o(\eta)$ (when $\eta \rightarrow 0$ ) the formula (73) at $\lambda=r_{n, l}$ gives $\left(r_{n, l}-s_{\check{\mathbf{q}}_{l}}\right)\left(\lambda+s_{-\check{\mathrm{q}}_{l}}\right) \sim 2 \mathrm{i} B\left(\check{\mathbf{q}}_{l}\right) r_{n, l} / A\left(\check{\mathrm{q}}_{l}\right)$. Therefore

$$
\frac{-s_{\check{\mathbf{q}}_{l}}+s_{-\check{\mathrm{q}}_{l}}}{\left(r_{n, l}-s_{\check{\mathbf{q}}_{l}}\right)\left(r_{n, l}+s_{-\check{\mathrm{q}}_{l}}\right)} \rightarrow \frac{1}{r_{n, l}} \quad \text { for } \eta \rightarrow 0
$$

for the corresponding factor in the formula (56) for norm. Note that this result is independent of which of the two quasi-momenta $q_{n, l}$ or $-q_{n, l}$ is excited. Now we have to take the product of one term (56) for R with another for NS. Since the terms at $s_{\pi}$ appear only in NS (R) for $n$ odd (even), we get two slightly different formulas for these cases:

For $n$ odd we have

$$
\begin{aligned}
& \frac{\left\langle\Phi_{0} \mid \Phi_{0}\right\rangle\left\langle\Phi_{1} \mid \Phi_{1}\right\rangle}{\left\langle\tilde{\Psi}_{0, \mathbf{0}} \mid \tilde{\Psi}_{0, \mathbf{0}}\right\rangle^{2}}=2^{|\mathcal{D}|} \prod_{k=1}^{n-1}\left(2 r_{n, k}\right) \cdot \frac{\prod_{k-\text { odd }}\left(\lambda_{\pi} \pm r_{n, k}\right)}{\prod_{k-\text { even }}\left(\lambda_{\pi}+r_{n, k}\right)} \cdot \frac{\prod_{k-\text { even }}\left(\lambda_{0} \pm r_{n, k}\right)}{\prod_{k-\text { odd }}\left(\lambda_{0}+r_{n, k}\right)} \times \\
& \times \frac{\prod_{k<l, k, l-\text { odd }}\left(\left(r_{n, k}+r_{n, l}\right)\left( \pm r_{n, k} \pm r_{n, l}\right)\right) \prod_{k<l, k, l-\text { even }}\left(\left(r_{n, k}+r_{n, l}\right)\left( \pm r_{n, k} \pm r_{n, l}\right)\right)}{\prod_{k-\text { odd }, l \text {-even }}\left(\left( \pm r_{n, k}+r_{n, l}\right)\left(r_{n, k} \pm r_{n, l}\right)\right)}
\end{aligned}
$$

and for $n$ even

$$
\begin{aligned}
& \frac{\left\langle\Phi_{0} \mid \Phi_{0}\right\rangle\left\langle\Phi_{1} \mid \Phi_{1}\right\rangle}{\left\langle\tilde{\Psi}_{0, \mathbf{0}} \mid \tilde{\Psi}_{0, \mathbf{0}}\right\rangle^{2}}=2^{|\mathcal{D}|} \prod_{k=1}^{n-1}\left(2 r_{n, k}\right) \cdot\left(\lambda_{0}+\lambda_{\pi}\right) \frac{\prod_{k-\text { even }}\left(\lambda_{0} \pm r_{n, k}\right)\left(\lambda_{\pi} \pm r_{n, k}\right)}{\prod_{k-\text { odd }}\left(\lambda_{0}+r_{n, k}\right)\left(\lambda_{\pi}+r_{n, k}\right)} \times \\
& \times \frac{\prod_{k<l, k, l-\text { odd }}\left(\left(r_{n, k}+r_{n, l}\right)\left( \pm r_{n, k} \pm r_{n, l}\right)\right) \prod_{k<l, k, l-\text { even }}\left(\left(r_{n, k}+r_{n, l}\right)\left( \pm r_{n, k} \pm r_{n, l}\right)\right)}{\prod_{k-\text { odd }, l \text {-even }}\left(\left( \pm r_{n, k}+r_{n, l}\right)\left(r_{n, k} \pm r_{n, l}\right)\right)}
\end{aligned}
$$

where the sign at $\pm r_{n, m}$ is fixed ' - ' if $m \in \mathcal{D}$ and ' + ' otherwise.

The final result (71) will be given in terms of $\lambda_{0}, \lambda_{\pi}$ and $r_{n, k}, k=1,2, \ldots, n-1$. Now if the eigenvalue polynomials contain the factors $\left(\lambda-r_{n, l}\right)^{2}$ instead of $\left(\lambda+r_{n, l}\right)^{2}$ for some $l$ we have to replace $r_{n, l} \rightarrow-r_{n, l}$ in the final formula for the matrix element (71) for all such $l$.

Let us finish this section by giving as an example the matrix element for the 3-site chain between $\left|\Phi_{0}\right\rangle=|\pi, \pi / 3\rangle_{\mathrm{NS}}$ and $\left|\Phi_{1}\right\rangle=|0\rangle_{\mathrm{R}}$. Using (74) we have

$$
\frac{\left\langle\Phi_{0} \mid \Phi_{0}\right\rangle\left\langle\Phi_{1} \mid \Phi_{1}\right\rangle}{\left\langle\tilde{\Psi}_{0, \mathbf{0}} \mid \tilde{\Psi}_{0, \mathbf{0}}\right\rangle^{2}}=-\frac{8 r_{31} r_{32}\left(r_{31}+s_{\pi}\right)\left(r_{32}-s_{0}\right)}{\left(r_{32}^{2}-r_{31}^{2}\right)\left(r_{31}-s_{0}\right)\left(r_{32}-s_{\pi}\right)} .
$$

Since in this case $\mathcal{D}=\{1\}$, formula (170) gives

$$
\frac{\left\langle\Phi_{0}\left|\mathbf{u}_{3}\right| \Phi_{1}\right\rangle\left\langle\Phi_{1}\left|\mathbf{u}_{3}\right| \Phi_{0}\right\rangle}{\left\langle\tilde{\Psi}_{0, \mathbf{0}} \mid \tilde{\Psi}_{0, \mathbf{0}}\right\rangle^{2}}=-\frac{2 r_{31}\left(s_{0}+s_{\pi}\right)}{\left(r_{31}-s_{0}\right)\left(r_{31}-s_{\pi}\right)} .
$$


Finally,

$$
\frac{\left\langle\Phi_{0}\left|\mathbf{u}_{3}\right| \Phi_{1}\right\rangle\left\langle\Phi_{1}\left|\mathbf{u}_{3}\right| \Phi_{0}\right\rangle}{\left\langle\Phi_{0} \mid \Phi_{0}\right\rangle\left\langle\Phi_{1} \mid \Phi_{1}\right\rangle}=\frac{\left(s_{0}+s_{\pi}\right)\left(s_{2 \pi / 3}^{2}-s_{\pi / 3}^{2}\right)\left(s_{2 \pi / 3}-s_{\pi}\right)}{4 s_{2 \pi / 3}\left(s_{\pi / 3}^{2}-s_{\pi}^{2}\right)\left(s_{2 \pi / 3}-s_{0}\right)},
$$

where due to (65)

$$
s_{\pi / 3}^{2}=r_{31}^{2}=\frac{b^{4}-b^{2}+1}{a^{4}-a^{2}+1}, \quad s_{2 \pi / 3}^{2}=r_{32}^{2}=\frac{b^{4}+b^{2}+1}{a^{4}+a^{2}+1} .
$$

\section{Conclusions}

In this paper we continue our calculation of state vectors and matrix elements of the finite-size inhomogenous $\mathbb{Z}_{N}$-Baxter-Bazhanov-Stroganov lattice spin model, using the method of Separation of Variables. In a previous paper [1] we gave the right eigenvectors for the auxiliary system and the Baxter equations which determine the periodic boundary condition BBS-eigenvectors. Here we complete this work by calculating explicit formulae for the corresponding left eigenvectors and norms and matrix elements of a single operator. A main result is the expression of Theorem 1 for the norm of the state vectors of the auxiliary system: the multiple summation over the intermediate states is performed, so that the norm is put into a factorized form. For $N=2$ the Baxter equations are solved explicitly, and also the norm of the periodic model is put into a compact factorized form. Since it is an open task to perform the intermediate spin summations for matrix elements of the inhomogenous periodic model, in the last

Section we proceeded with the homogenous Ising model parameters. There we manage to perform the summations and we present a new factorized formula for the matrix elements of spin operator, delegating the somewhat lengthy derivation and comparison with the corresponding formula in [14, 15] to our sequel paper [16].

\section{Acknowledgements}

This work has been supported by the Heisenberg-Landau program HLP-2007. SP was supported by the RFBR grant 05-01-01086 and grant for support of scientific schools NSh-8065.2006.2, NI and VS were supported by INTAS grant 05-1000008-7865 and Ukrainian DFFD grant. GvG is grateful to the Department of Physics of Complex Systems of the Weizmann Institute for kind hospitality.

\section{Appendix: The main summation formula}

Consider a degree $n$ polynomial $f(t)=f_{n} t^{n}+\ldots+f_{0}$ and its interpolation formula through $n+1$ arbitrary not coinciding points $c_{1}, \ldots, c_{n+1}$ :

$$
f(t)=\sum_{k=1}^{n+1} f\left(c_{k}\right) \prod_{s \neq k}^{n+1} \frac{t-c_{s}}{c_{k}-c_{s}}, \quad f_{n}=\sum_{k=1}^{n+1} \frac{f\left(c_{k}\right)}{\prod_{s \neq k}^{n+1}\left(c_{k}-c_{s}\right)} .
$$


We shall use (76) for $f_{n}=0$, i.e. for polynomials $f(t)$ of degree less than $n$, obtaining a sum rule valid for an arbitrary choice of the parameters $\left\{c_{1}, \ldots, c_{n+1}\right\}$ :

$$
\sum_{k=1}^{n+1} \frac{f\left(c_{k}\right)}{\prod_{s \neq k}^{n+1}\left(c_{k}-c_{s}\right)}=0
$$

Now take an arbitrary polynomial $f(t)$ of degree less than $n+N$ and choose $n+N$ points $\left(c_{1}, \ldots, c_{n+N}\right)=\left(b_{1}, \ldots, b_{n}, r, \omega r, \ldots, \omega^{N-1} r\right)$. Then the sum rule corresponding to (77) is

$$
\sum_{\rho=0}^{N-1} \frac{\omega^{\rho} r f\left(\omega^{\rho} r\right)}{\prod_{s=1}^{n}\left(\omega^{\rho} r-b_{s}\right)}=N r^{N} \sum_{k=1}^{n} \frac{f\left(b_{k}\right)}{\left(r^{N}-b_{k}^{N}\right) \prod_{\substack{s=1 \\ s \neq k}}^{n}\left(b_{k}-b_{s}\right)}
$$

since $\prod_{i=0, i \neq \rho}^{N-1}\left(\omega^{\rho} r-\omega^{i} r\right)=N \omega^{-\rho} r^{N-1}$.

The goal of this Appendix is to prove equation (28) of the main text, i.e. we shall show by induction that for the sets $\mathbf{a}^{(n)}=\left\{a_{1}, \ldots, a_{n-2}\right\}=\left\{r_{1} \omega^{-\rho_{1}}, \ldots, r_{n-2} \omega^{-\rho_{n-2}}\right\}$

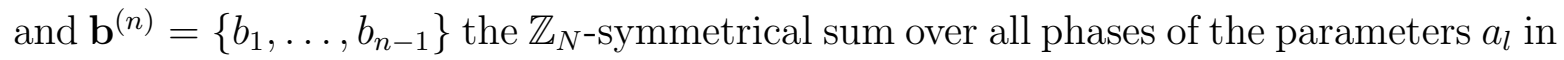

$$
S_{n}\left(\mathbf{a}^{(n)}, \mathbf{b}^{(n)}\right)=\sum_{\rho_{1}, \ldots, \rho_{n-2} \in\left(\mathbb{Z}_{N}\right)^{n-2}} \prod_{l=1}^{n-2}\left(a_{l} \frac{\prod_{j=l+1}^{n-2}\left(a_{l}-a_{j}\right)}{\prod_{k=1}^{n-1}\left(a_{l}-b_{k}\right)}\right)
$$

can be performed explicitly, so that we obtain (179) in the factorized form

$$
S_{n}\left(\mathbf{a}^{(n)}, \mathbf{b}^{(n)}\right)=N^{n-2} \prod_{l=1}^{n-2} a_{l}^{N} \prod_{k<k^{\prime}}^{n-1} \frac{b_{k}^{N}-b_{k^{\prime}}^{N}}{b_{k}-b_{k^{\prime}}} \frac{\prod_{l<l^{\prime}}^{n-2}\left(a_{l}^{N}-a_{l^{\prime}}^{N}\right)}{\prod_{l=1}^{n-1} \prod_{l^{\prime}=1}^{n-2}\left(b_{l}^{N}-a_{l^{\prime}}^{N}\right)} .
$$

This assertion is correct for $n=3$, as can be seen by explicit summation, using $\sum_{\rho \in \mathbb{Z}_{N}} b /\left(r \omega^{-\rho}-b\right)=N b^{N} /\left(r^{N}-b^{N}\right)$ :

$$
\begin{aligned}
S_{3}\left(a_{1} ; b_{1}, b_{2}\right) & =\sum_{\rho_{1}} \frac{a_{1}}{\left(a_{1}-b_{1}\right)\left(a_{1}-b_{2}\right)}=\sum_{\rho_{1}} \frac{1}{b_{1}-b_{2}}\left(\frac{b_{2}}{b_{2}-r_{1} \omega^{-\rho_{1}}}-\frac{b_{1}}{b_{1}-r_{1} \omega^{-\rho_{1}}}\right) \\
& =\frac{N}{b_{1}-b_{2}}\left(\frac{b_{2}^{N}}{b_{2}^{N}-a_{1}^{N}}-\frac{b_{1}^{N}}{b_{1}^{N}-a_{1}^{N}}\right)=\frac{N a_{1}^{N}\left(b_{1}^{N}-b_{2}^{N}\right)}{\left(b_{1}-b_{2}\right)\left(b_{1}^{N}-a_{1}^{N}\right)\left(b_{2}^{N}-a_{1}^{N}\right)} .
\end{aligned}
$$

For general $n$ it will be useful to use the induction assumption (179) also in the form

$$
S_{n}\left(\mathbf{a}^{(n)}, \mathbf{b}^{(n)}\right)=\tilde{S}_{n}\left(\mathbf{a}^{(n)}\right) \prod_{k<k^{\prime}}^{n-1} \frac{b_{k}^{N}-b_{k^{\prime}}^{N}}{b_{k}-b_{k^{\prime}}} \frac{1}{\prod_{l=1}^{n-1} \prod_{l^{\prime}=1}^{n-2}\left(b_{l}^{N}-a_{l^{\prime}}^{N}\right)}
$$

with

$$
\tilde{S}_{n}\left(\mathbf{a}^{(n)}\right)=N^{n-2}\left(\prod_{l=1}^{n-2} a_{l}^{N}\right) \prod_{l<l^{\prime}}^{n-2}\left(a_{l}^{N}-a_{l^{\prime}}^{N}\right) .
$$

In proceeding with the induction proof of (80) we start separating terms involving the last summation (over $\rho_{n-2}$ ) in (779) and perform this sum using (78) with $f(t)=$ $\prod_{m=1}^{n-3}\left(a_{m}-t\right)$ :

$$
\begin{aligned}
S_{n} & =\sum_{\rho_{1}, \ldots, \rho_{n-3}}\left(\prod_{l=1}^{n-3} a_{l} \frac{\prod_{j=l+1}^{n-3}\left(a_{l}-a_{j}\right)}{\prod_{m=1}^{n-1}\left(a_{l}-b_{m}\right)}\right) \sum_{\rho_{n-2}} a_{n-2} \frac{\prod_{m=1}^{n-3}\left(a_{m}-a_{n-2}\right)}{\prod_{s=1}^{n-1}\left(a_{n-2}-b_{s}\right)} \\
& =\sum_{\rho_{1}, \ldots, \rho_{n-3}}\left(\prod_{l=1}^{n-3} a_{l} \frac{\prod_{j=l+1}^{n-3}\left(a_{l}-a_{j}\right)}{\prod_{m=1}^{n-1}\left(a_{l}-b_{m}\right)}\right) N a_{n-2}^{N} \sum_{k=1}^{n-1} \frac{\prod_{m=1}^{n-3}\left(a_{m}-b_{k}\right)}{\left(a_{n-2}^{N}-b_{k}^{N}\right) \prod_{\substack{n=1 \\
s=1 \\
s \neq k}}^{n-1}\left(b_{k}-b_{s}\right)} .
\end{aligned}
$$


Form-factors in the BBS model I: Norms and matrix elements

The first summation in the last equation involves also the phases of $a_{m}$ in the term $\prod_{m=1}^{n-3}\left(a_{m}-b_{k}\right)$ of the last numerator. However, this term cancels just the term $m=k$ in the denominator of the first bracket. So, comparing to (79) for $n \rightarrow n-1$ we can write

$$
S_{n}\left(\mathbf{a}^{(n)}, \mathbf{b}^{(n)}\right)=N a_{n-2}^{N} \sum_{k=1}^{n-1} \frac{S_{n-1}\left(\mathbf{a}_{n-2}^{(n)}, \mathbf{b}_{k}^{(n)}\right)}{\left(a_{n-2}^{N}-b_{k}^{N}\right) \prod_{\substack{n=1 \\ s \neq 1 \\ s \neq k}}^{n-1}\left(b_{k}-b_{s}\right)},
$$

where $\mathbf{b}_{k}^{(n)}$ is the $n-2$ component vector resulting from $\mathbf{b}^{(n)}$ omitting the component $b_{k}$. Similarly, $\mathbf{a}_{n-2}^{(n)}$ is the $n-3$-component vector resulting from $\mathbf{a}^{(n)}$ by omitting the component $a_{n-2}$. Now we can use the induction assumption (80) to insert here $S_{n-1}$ :

$$
\begin{aligned}
& S_{n}\left(\mathbf{a}^{(n)}, \mathbf{b}^{(n)}\right)= N a_{n-2}^{N} \sum_{k=1}^{n-1} \frac{1}{\left(a_{n-2}^{N}-b_{k}^{N}\right) \prod_{\substack{n=1 \\
s=1 \\
s \neq k}}^{n-1}\left(b_{k}-b_{s}\right)} \times \\
& \times N^{n-3}\left(\prod_{l=1}^{n-3} a_{l}^{N}\right) \prod_{\substack{m<m^{\prime} \\
m, m^{\prime} \neq k}}^{n-1} \frac{b_{m}^{N}-b_{m^{\prime}}^{N}}{b_{m}-b_{m^{\prime}}} \frac{\prod_{l<l^{\prime}}^{n-3}\left(a_{l}^{N}-a_{l^{\prime}}^{N}\right)}{\prod_{\substack{l=1 \\
l \neq k}}^{n-1} \prod_{l^{\prime}=1}^{n-3}\left(b_{l}^{N}-a_{l^{\prime}}^{N}\right)} .
\end{aligned}
$$

It is more convenient to write (82) in terms of $\tilde{S}_{n}\left(\mathbf{a}^{(n)}\right)$, equation (81), since then the b-dependence is explicit:

$$
\begin{aligned}
\tilde{S}_{n}\left(\mathbf{a}^{(n)}\right) & \prod_{k^{\prime}<k^{\prime \prime}}^{n-1} \frac{b_{k^{\prime}}^{N}-b_{k^{\prime \prime}}^{N}}{b_{k^{\prime}}-b_{k^{\prime \prime}}} \frac{1}{\prod_{l=1}^{n-1} \prod_{l^{\prime}=1}^{n-2}\left(b_{l}^{N}-a_{l^{\prime}}^{N}\right)}=N a_{n-2}^{N} \tilde{S}_{n-1}\left(\mathbf{a}_{n-2}^{(n)}\right) \times \\
& \times \sum_{k=1}^{n-1} \prod_{\substack{k^{\prime}<k^{\prime \prime} \\
k^{\prime}, k^{\prime \prime} \neq k}}^{n-1} \frac{b_{k^{\prime}}^{N}-b_{k^{\prime \prime}}^{N}}{b_{k^{\prime}}-b_{k^{\prime \prime}}} \frac{1}{\prod_{\substack{l=1 \\
l \neq k}}^{n-1} \prod_{l^{\prime}=1}^{n-3}\left(b_{l}^{N}-a_{l^{\prime}}^{N}\right)} \frac{1}{\left(a_{n-2}^{N}-b_{k}^{N}\right) \prod_{\substack{s=1 \\
s \neq k}}^{n-1}\left(b_{k}-b_{s}\right)} .
\end{aligned}
$$

Apart from a sign, the terms linear in the $b_{k^{\prime}}$ cancel since

$$
\frac{\prod_{k^{\prime}<k^{\prime \prime}}^{n-1}\left(b_{k^{\prime}}-b_{k^{\prime \prime}}\right)}{\prod_{\substack{k^{\prime}<k^{\prime \prime} \\ k^{\prime}, k^{\prime \prime} \neq k}}^{n-1}\left(b_{k^{\prime}}-b_{k^{\prime \prime}}\right)}=\prod_{k^{\prime}=k+1}^{n-1}\left(b_{k}-b_{k^{\prime}}\right) \prod_{k^{\prime}=1}^{k-1}\left(b_{k^{\prime}}-b_{k}\right)=(-1)^{k-1} \prod_{s=1, s \neq k}^{n-1}\left(b_{k}-b_{s}\right) \text {. }
$$

Using the same formula for the $b_{k^{\prime}}^{N}$-terms and simplifying the $b_{l}^{N}-a_{l^{\prime}}^{N}$-terms, we get

$$
\tilde{S}_{n}\left(\mathbf{a}^{(n)}\right)=-N a_{n-1}^{N} \tilde{S}_{n-1}\left(\mathbf{a}_{n-2}^{(n)}\right) \sum_{k=1}^{n-1} \frac{\prod_{l=1}^{n-3}\left(b_{k}^{N}-a_{l}^{N}\right) \prod_{l \neq k}^{n-1}\left(b_{l}^{N}-a_{n-2}^{N}\right)}{\prod_{s=1, s \neq k}^{n-1}\left(b_{k}^{N}-b_{s}^{N}\right)} .
$$

Here the sum can be calculated using the identity

$$
\sum_{k=1}^{n-1} \prod_{l=1}^{n-3}\left(b_{k}^{N}-a_{l}^{N}\right) \prod_{\substack{s=1 \\ s \neq k}}^{n-1} \frac{b_{s}^{N}-a_{n-2}^{N}}{b_{k}^{N}-b_{s}^{N}}=-\prod_{l=1}^{n-3}\left(a_{l}^{N}-a_{n-2}^{N}\right)
$$

so that

$$
\tilde{S}_{n}\left(\mathbf{a}^{(n)}\right)=N a_{n-2}^{N} \tilde{S}_{n-1}\left(\mathbf{a}_{n-2}^{(n)}\right) \prod_{l=1}^{n-3}\left(a_{l}^{N}-a_{n-2}^{N}\right)
$$

which confirms (81) and so also (80). 


\section{References}

[1] von Gehlen G, Iorgov N, Pakuliak S and Shadura V 2006 The Baxter-Bazhanov-Stroganov model: separation of variables and the Baxter equation J. Phys. A: Math. Gen. 39 7257-7282 (Preprint arXiv:nlin.SI/0603028)

[2] Baxter R J 2004 Transfer matrix functional relations for the generalized $\tau_{2}\left(t_{q}\right)$ model J. Stat. Phys. 117 1-25

[3] Baxter R J 1989 Superintegrable chiral Potts model: Thermodynamic properties, an "Inverse" model, and a simple associated Hamiltonian J. Stat. Phys. 57 1-39

[4] Bazhanov V V and Stroganov Yu G 1990 Chiral Potts model as a descendant of the six-vertex model J. Stat. Phys. 59 799-817

[5] Baxter R J, Bazhanov V V and Perk J H H 1990 Functional relations for the transfer matrices of the Chiral Potts model, Intern. J. Mod. Phys. B 4 803-869

[6] Baxter R J 1990 Chiral Potts model: eigenvalues of the transfer matrix Phys. Lett. A146 110-114

[7] Korepanov I G 1987 Hidden symmetries in the 6-vertex model, Chelyabinsk Polytechnical Institute, archive VINITI No. 1472-V87 (in Russian);

1994 Hidden symmetries in the 6-vertex model of Statistical Physics Zapiski Nauchn. Semin. POMI 215 163-177 (Preprint hep-th/9410066);

The method of vacuum vectors in the theory of Yang-Baxter equation Preprint nlin.SI/0010024

[8] Roan S S 2007 Fusion operators in the generalized $\tau^{(2)}$-model and root-of-unity smmetry of the XXZ spin chain of higher spin J. Phys. A: Math. Gen. 40 1481-1511

[9] Sklyanin E K 1990 Functional Bethe Ansatz in Integrable and Superintegrable Systems, ed. Kupershmidt B A, World Scientific, Singapore, 8-33

[10] Bugrij A I, Iorgov N Z and Shadura V N 2005 Alternative Method of Calculating the Eigenvalues of the Transfer Matrix of the $\tau_{2}$ Model for $N=2$ JETP Lett. $82311-315$

[11] Tarasov V O 1992 Cyclic monodromy matrices for the R-matrix of the six-vertex model and the chiral Potts model with fixed spin boundary conditions Intern. J. Mod. Phys. A7 Suppl. 1B $963-975$

[12] Kharchev S and Lebedev D 2000 Eigenfunctions of $G L(N, \mathbb{R})$ Toda chain: The Mellin-Barnes representation JETP Lett. $71235-238$

[13] Babelon O 2003 Equations in dual variables for Whittaker functions, Lett. Math. Phys. 65 229-240

[14] Bugrij A and Lisovyy O 2003 Spin matrix elements in 2D Ising model on the finite lattice, Phys. Lett. A 319 390-394 (Preprint arXiv:0708.3625 [nlin.SI])

[15] Bugrij A and Lisovyy O 2004 Correlation Function of the Two-Dimensional Ising Model on a Finite Lattice: II, Theor. and Math. Physics 140 987-1000 (Preprint arXiv:0708.3643 [nlin.SI])

[16] von Gehlen G, Iorgov N, Pakuliak S, Shadura V anf Tykhyy Yu 2007 Form-factors in the finite size Baxter-Bazhanov-Stroganov model II. Ising model on the finite lattice. In preparation.

[17] Bazhanov V V and Baxter R J 1992 New solvable lattice models in three dimensions J. Stat. Phys. 69 453-485

[18] Lisovyy O 2006 Transfer matrix eigenvectors of the Baxter-Bazhanov-Stroganov $\tau_{2}$-model for $N=2$ J. Phys. A: Math. Gen. 39 2265-2285 (Preprint arXiv:nlin.SI/0512026) 